\title{
DYNAMIC APPROACHES FOR SOME TIME-INCONSISTENT OPTIMIZATION PROBLEMS
}

\author{
BY CHANDRASEKHAR KARNAM, Jin MA ${ }^{1}$ AND JIANFENG ZHANG ${ }^{2}$ \\ University of Southern California
}

\begin{abstract}
In this paper, we investigate possible approaches to study general timeinconsistent optimization problems without assuming the existence of optimal strategy. This leads immediately to the need to refine the concept of time consistency as well as any method that is based on Pontryagin's maximum principle. The fundamental obstacle is the dilemma of having to invoke the Dynamic Programming Principle (DPP) in a time-inconsistent setting, which is contradictory in nature. The main contribution of this work is the introduction of the idea of the "dynamic utility" under which the original timeinconsistent problem (under the fixed utility) becomes a time-consistent one. As a benchmark model, we shall consider a stochastic controlled problem with multidimensional backward SDE dynamics, which covers many existing time-inconsistent problems in the literature as special cases; and we argue that the time inconsistency is essentially equivalent to the lack of comparison principle. We shall propose three approaches aiming at reviving the DPP in this setting: the duality approach, the dynamic utility approach and the master equation approach. Unlike the game approach in many existing works in continuous time models, all our approaches produce the same value as the original static problem.
\end{abstract}

1. Introduction. In this paper, we propose some possible approaches to tackle the general time-inconsistent optimization problems in continuous time setting. These approaches are different from all the existing ones in the literature, and are based on our new understanding of the time inconsistency. We note that the time inconsistency appears naturally and frequently in economics and finance (see, e.g., Kydland-Prescott [25] and Kahneman-Tversky [22, 23]). We refer to the frequently cited survey by Strotz [32] for the fundamentals of this problem, and Zhou [36] for some recent development on continuous time models. We should point out that it was [36] that brought the time inconsistency issue to our attention.

I. Time inconsistency. We begin by briefly describing the time inconsistency in an optimization problem that has been understood so far. Consider an optimization

Received April 2016; revised January 2017.

${ }^{1}$ Supported in part by NSF Grant 1106853.

${ }^{2}$ Supported in part by NSF Grant 1413717.

MSC2010 subject classifications. 49L20, 60H10, 91C99, 91G80, 35R15.

Key words and phrases. Time inconsistency, dynamic programming principle, stochastic maximum principle, comparison principle, duality, dynamic utility, master equation, path derivative. 
problem over a time interval $[0, T]$ :

$$
V_{0}:=\sup _{u \in \mathscr{U}[0, T]} J(u),
$$

where $\mathscr{U}_{[0, T]}$ is an appropriate set of admissible controls $u$ defined on $[0, T]$, and $J(u)$ is a certain utility functional associated to $u$. Clearly, the problem (1.1) is static. Its dynamic counterpart is the following optimization problem over $[t, T]$, for any $t \in[0, T]$ :

$$
V_{t}:=\operatorname{esssup}_{u \in \mathscr{U}_{[t, T]}} J_{t}(u)
$$

Here, $\mathscr{U}_{[t, T]}$ is the corresponding set of admissible controls on $[t, T]$ and the utility functional $J_{t}$ usually involves some conditional expectation, and thus could be random.

An admissible control $u^{*} \in \mathscr{U}_{[0, T]}$ is called "optimal" for the problem (1.1) if $J\left(u^{*}\right)=V_{0}$. Defining optimal control $u^{t, *}$ for the problem (1.2) similarly and assuming their existence, we say the problem (1.2) is time consistent if, for any $t \in[0, T]$, it holds that

$$
u_{s}^{t, *}=u_{s}^{*}, \quad t \leq s \leq T .
$$

The relation (1.3) amounts to saying that a (temporally) global optimum must be a local one. The optimization problem (1.2) is called time inconsistent if (1.3) fails to hold. Intuitively, time inconsistency means an optimal strategy today may not be optimal tomorrow.

Since the early work [32], there have been typically two approaches for treating the time-inconsistent problems, both focusing on the optimal control: (i) the strategy of precommitment, and (ii) the strategy of consistent planning. The former is to solve the static optimization problem (1.1), and then simply insist on using $u^{*}$ (assuming it exists) throughout $[0, T]$, despite the fact that it may not be optimal anymore when $t>0$. The latter one has developed into the popular "game approach" in the literature, in which the player plays with infinitely many future selves. To illustrate the idea, let us consider the discrete time setting: $0=t_{0}<\cdots<t_{n}=T$. The "consistent planning" amounts to saying that at any $t_{i}$, the player tries to find optimal strategy $u$ on $\left[t_{i}, t_{i+1}\right)$ by assuming the future selves have already found the optimal strategies and will actually use them on $\left[t_{i+1}, T\right]=\left[t_{i+1}, t_{i+2}\right) \cup \cdots \cup\left[t_{n-1}, T\right]$. We note that an equilibrium in such a game approach should be similar to that of a principal agent problem, that is, in the sense of a sequential optimization problem, rather than a Nash equilibrium.

The game approach makes sense in many applications, but is very challenging in continuous time setting (being a game with uncountably many players). There have been some successful applications of this approach in continuous time models; see, for example, Bjork-Murgoci [2], Ekeland-Lazrak [11], Hu-Jin-Zhou [20] and Yong [34], to mention a few. It is worth noting that since under the game 
framework the problem is time consistent, which enables one to apply the standard tools such as dynamic programming and HJB equations. However, typically the value of the game problem at $t=0$ is different from the original value $V_{0}$ in (1.1) (unless the problem is time consistent), thus the solution of the game approach, even if it exists, does not really solve the problem (1.1).

In this paper, we will focus on the value $V_{0}$ of the original static problem (1.1). We would like to emphasize that the problem (1.1), or its "precommitment" nature, actually makes more sense in some applications. For example, in the so-called principal-agent problem (see Section 2.3 below), practically the principal cannot change the contract once it commenced (at least not as frequently as the game approach requires), therefore, one is obliged to follow the contract designed at $t=0$ for the whole contractual period. In fact, problem (1.1) is a mathematically interesting problem in its own right.

Another main feature of this paper is that, unlike most of the works in the "time inconsistency" literature to date, we shall remove the presumption of the existence of optimal strategy. In fact, as is well known in stochastic control literature, it is not unusual that the optimal control fail to exist. It has been noted, however, that without the optimal control (or equilibrium in game approach) it is not even clear how to define the notion of time consistency/inconsistency in most of the current literature. But on the other hand, the value $V_{0}$ is always well defined, regardless the existence of optimal control. Our main task is thus to find the new (time consistent) methods to solve the original value $V_{0}$ without using optimal controls, and to revive the dynamical programming method in a novel context.

II. Our main observation. It is well understood that there are typically two approaches to solve the optimization problem (1.1): the Dynamic Programming Principle (DPP for short) and the Stochastic Maximum Principle (SMP for short). The former relies fundamentally on the time consistency; whereas the latter requires the existence of optimal control. We then immediately find ourselves facing the dilemma: on the one hand the SMP, as a necessary condition, is no longer relevant without an optimal control; but on the other hand, DPP does not make sense either due to the lack of time consistency.

To "revive" the DPP for the static problem (1.1), our first plan is based on the following simple but crucial observation: the problem (1.2) is time inconsistent partially due to the fact that, modulus some conditional expectation, the utility $J_{t}$ in (1.2) is essentially the same as the utility $J$ in (1.1), which could be in conflict with the nature of the problem and causing the time inconsistency. Therefore, if we allow $J_{t}$ to vary more freely with the time $t$, denoting it by $J(t, u)$, then it is hopeful that the new dynamic optimization problem

$$
\tilde{V}_{t}:=\sup _{u \in \mathscr{U}[t, T]} J(t, u)
$$

could become time consistent with the right choice of $J(t, \cdot)$. In particular, if we require that $J(0, u)=J(u)$, then $\tilde{V}_{0}=V_{0}$ and we are indeed solving the original 
problem (1.1). In fact, as we will see in the next section, when the optimal control $u^{*}$ exists, one can easily construct such $J(t, \cdot)$ by utilizing the optimal $u^{*}$. The real challenge is, of course, to find a desired $J(t, \cdot)$ without using $u^{*}$ or in the situation where $u^{*}$ does not exist.

We remark that, given the initial value $J(0, u)=J(u)$, the dynamic $J(\cdot, \cdot)$ will be sought forwardly (in time), and thus it is in spirit similar to the notion of forward utility proposed in $[28,29]$. However, it should be emphasized that the forward utility $U(t, \cdot)$ in $[28,29]$ is applied on an optimization problem over time period $[0, t]$, while our utility $J(t, \cdot)$ is over time period $[t, T]$. Namely, there is a fundamental difference between the two notions.

Finally, we should point out that similar ideas of "dynamic utilities" have also appeared in the literature under various different context that are time inconsistent in nature; see, for example, Bouchard-Elie-Touzi [4], Cohen-Elliot [6], Cui-LiWang-Zhu [8], Feinstein-Rudloff [19] and Miller [27].

III. The proposed approaches. Our second main observation in this paper is that many time-inconsistent problems in the literature can be transformed into control problems on multidimensional (possibly infinite dimensional) forwardbackward SDEs (see Section 2 for details). Therefore, in what follows we shall focus on the following benchmark optimization problem for controlled multidimensional backward SDEs:

$$
V_{0}:=\sup _{u \in \mathscr{U}[0, T]} \varphi\left(Y_{0}^{u}\right)
$$

$$
\text { where } Y_{t}^{u}=\xi+\int_{t}^{T} f\left(s, Y_{s}^{u}, Z_{s}^{u}, u_{s}\right) d s-\int_{t}^{T} Z_{s}^{u} d B_{s}, \quad t \in[0, T] .
$$

We note that in (1.5) we have made two simplifications in order to focus more on the main issue of time inconsistency: the controlled dynamics is only a backward SDE and the dimension is finite. All the results in this paper can be extended to the controlled forward-backward SDE case, but with heavier presentations. We prefer not to seek such generality in this paper. The infinite dimensional case, however, is more challenging, and we shall leave it to future study.

We start with a "duality approach" by first noticing that

$$
V_{0}=\sup _{y \in \mathscr{D}_{0}} \varphi(y) \quad \text { where } \mathscr{D}_{0}:=\left\{Y_{0}^{u}: u \in \mathscr{U}_{[0, T]}\right\} .
$$

We shall argue that, in the Markovian case, the "reachable set" $\mathscr{D}_{0}$ can be written as

$$
\overline{\mathscr{D}}_{0}=\mathscr{N}(0,0):=\{y: W(0,0, y)=0\},
$$

where $\overline{\mathscr{D}}_{0}$ is the closure of $\mathscr{D}_{0}, W(t, x, y)$ is the unique viscosity solution to certain standard HJB equation, and $\mathscr{N}(0,0)$ is the the so-called "nodal set" of $W$. Assuming $\varphi$ is continuous, we can first solve the HJB equation for $W$, then compute its 
nodal set $\mathscr{N}(0,0)$, and finally solve a simple finite dimensional optimization problem:

$$
V_{0}=\sup _{y \in \mathscr{N}(0,0)} \varphi(y)
$$

We note that the idea of nodal set was used in Ma-Yong [26] for solving a forwardbackward SDE (without control $u$ ), and we call this a "duality approach." We shall further argue that the duality holds in non-Markovian case as well, by utilizing the viscosity theory of path dependent PDEs developed by Ekren-Keller-TouziZhang [12] and Ekren-Touzi-Zhang [13, 14].

While the duality approach is quite generally applicable under mild conditions, it solves only the static problem $V_{0}$. In particular, it does not provide a time consistent dynamic value $\tilde{V}_{t}$. Our next step is to extend the set $\mathscr{D}_{0}$ and the duality (1.7) to a dynamic version:

$$
\mathscr{D}_{t}:=\left\{Y_{t}^{u}: u \in \mathscr{U}_{[t, T]}\right\}, \quad \overline{\mathscr{D}_{t}}=\mathscr{N}\left(t, B_{t}\right):=\left\{y: W\left(t, B_{t}, y\right)=0\right\} .
$$

We shall argue that the family $\left\{\mathscr{D}_{t}\right\}_{0 \leq t \leq T}$ satisfies a geometric DPP, in the spirit of Soner-Touzi [31], and closely related to the set valued analysis (see, e.g., AubinFrankowska [1] and Feinstein-Rudloff [18]). However, we note that the following natural dynamic value:

$$
V_{t}:=\underset{u \in \mathscr{U}_{[t, T]}}{\operatorname{esssup}} \varphi\left(Y_{t}^{u}\right)=\underset{y \in \mathscr{D}_{t}}{\operatorname{esssup}} \varphi(y)=\operatorname{esssup}_{y \in \mathscr{N}\left(t, B_{t}\right)} \varphi(y)
$$

is typically time inconsistent. Here, $\operatorname{esssup}_{y \in \mathscr{D}_{t}} \varphi(y)$ means $\operatorname{esssup}_{y \in \mathbb{R}^{d}}[\varphi(y) \times$ $\left.\mathbf{1}_{\mathscr{D}_{t}}(y)\right]$, and the same definition also applies to other similar notation. The goal of our second approach is to find a dynamic utility function $\Phi(t, y)$ (possibly random) satisfying $\Phi(0, \cdot)=\varphi$ and that

$$
\tilde{V}_{t}:=\operatorname{esssup}_{u \in \mathscr{U}_{[t, T]}}^{\operatorname{en}} \Phi\left(t, Y_{t}^{u}\right)=\underset{y \in \mathscr{D}_{t}}{\operatorname{esssup}} \Phi(t, y)=\operatorname{esssup}_{y \in \mathscr{N}\left(t, B_{t}\right)} \Phi(t, y)
$$

is time consistent. We shall name this the "dynamic utility approach" for simplicity. An important observation coming out from the study of this approach is that the time inconsistency of (1.10) is essentially equivalent to the lack of comparison principle for the multidimensional BSDE, a well-known fact in BSDE theory. Thus our task becomes to find some dynamic utility function $\Phi(t, \cdot)$, which satisfies a certain comparison principle. In this paper, we succeed in finding a desired $\Phi$ in a linear case, and we shall leave the general nonlinear case, which seems to be quite challenging, to future research.

Our last approach borrows the idea from the mean field game literature (see, e.g., Cardaliaguet-Delarue-Lasry-Lions [5]), which we now describe. First, note that the value $V_{0}$ in (1.6) is clearly a function of the terminal condition $\xi$. Thus, for any $t \in[0, T]$ and random variable $\eta \in \mathbb{L}^{2}\left(\mathcal{F}_{t}\right)$, we define

$$
\Psi(t, \eta):=\sup _{u \in \mathscr{U}_{[0, t]}} \varphi\left(\mathscr{Y}_{0}^{u}(t, \eta)\right)
$$


where $\mathscr{Y}^{u}(t, \eta)$ is the solution to $\operatorname{BSDE}(1.5)$ on $[0, t]$, satisfying $\mathscr{Y}_{t}^{u}(t, \eta)=\eta$. Clearly, $\Psi(0, y)=\varphi(y)$ and $V_{0}=\Psi(T, \xi)$, thus both functions $\Phi$ in (1.11) and $\Psi$ in (1.12) are temporally "dynamic" in nature, with the same initial value $\varphi$. The main difference, however, is that in (1.11) the control is over $[t, T]$, whereas in (1.12) the control is over $[0, t]$. One should also note that, unlike in mean field theory where the functions often depend only on the laws of the random variables, the function $\Psi$ in (1.12) depends indeed on the random variable $\eta$, or more precisely on the joint law of $(\eta, B)$.

A very pleasant surprise of the (forward) value function $\Psi$ is that it satisfies the following form of DPP almost automatically, and can thus be viewed as time consistent:

$$
\begin{aligned}
& \Psi\left(t_{2}, \eta\right):=\sup _{u \in \mathscr{U}\left[t_{1}, t_{2}\right]} \Psi\left(t_{1}, \mathscr{Y}_{t_{1}}^{u}\left(t_{2}, \eta\right)\right), \quad \eta \in \mathbb{L}^{2}\left(\mathcal{F}_{t_{2}}\right), \\
& \text { for any } 0 \leq t_{1}<t_{2} \leq T .
\end{aligned}
$$

We shall emphasize that, unlike the usual DPP in stochastic control literature, (1.13) is forward (in time), that is, $t_{1}<t_{2}($ !). This is due to the fact that we are optimizing a backward controlled problem. To the best of our knowledge, such type of forward DPP is new.

Having obtained the DPP (1.13), we believe that certain HJB types of differential equations (for $\Psi$ ) should naturally come into the picture, which we shall name as the master equation, due to the nature of the function $\Psi$. We expect two features for this master equation: first, it should be a first-order partial differential equation in a certain sense, due to the forward nature of the DPP; second, it should involve certain path derivatives of $\eta$ in the sense of Dupire [10], due to the progressive measurability of $\Psi$ and the requirement $\eta$ being $\mathcal{F}_{t}$-measurable. We shall argue that when the function $\Psi$ defined by (1.12) is smooth (to be specified in the paper), it will be the unique (classical) solution to our master equation. The main difficulty of this approach, however, is when $\Psi$ does not have the desired smoothness. It then becomes a very interesting, albeit challenging, problem to propose appropriate notion of weaker solution to the master equation. We shall leave this to future research.

The rest of the paper is organized as follows. In Section 2, we present several examples of time-inconsistent problems. In Section 3, we introduce our model and explain the role of comparison principle in time-consistency issue. In Sections 4-6, we propose the three approaches, respectively.

2. Preliminaries and examples. Throughout this paper, we shall use the following canonical setup. Let $T>0$ be a fixed time horizon, $\Omega:=\{\omega \in$ $\left.C\left([0, T], \mathbb{R}^{d}\right): \omega_{0}=0\right\}$ the canonical space, $\mathcal{F}:=\mathscr{B}(\Omega)$, the Borel $\sigma$-filed of $\Omega$ and $\mathbb{P}_{0}$ the Wiener measure. Further, we let $B_{t}(\omega):=\omega_{t}, \omega \in \Omega$ be the canonical process and $\mathbb{F}:=\mathbb{F}^{B}$ the natural filtration generated by $B$, augmented by 
$\mathbb{P}_{0}$. Then $B$ is an $\mathbb{F}$-Brownian motion under $\mathbb{P}_{0}$. We also denote $\mathbb{E}:=\mathbb{E}^{\mathbb{P}_{0}}$ and $\mathbb{E}_{t}[\cdot]:=\mathbb{E}^{\mathbb{P}_{0}}\left[\cdot \mid \mathcal{F}_{t}\right]$ for simplicity, when the context is clear, and $\Lambda:=[0, T] \times \Omega$.

For a generic Euclidean space $\mathbb{X}$, we denote its inner product by $(x, y)=x \cdot y=$ $x^{\top} y$, its norm by $|x|:=(x, x)^{1 / 2}$ and its Borel $\sigma$-field by $\mathscr{B}(\mathbb{X})$. If $\mathbb{X}=\mathbb{R}^{d_{1} \times d_{2}}$, we denote $A: B:=\operatorname{tr}\left(A^{\top} B\right)$, for $A, B \in \mathbb{X}$. Also, let $\mathcal{G} \subseteq \mathcal{F}$ be any sub- $\sigma$-field and $[s, t] \subseteq[0, T]$, we denote:

- $\mathbb{L}^{2}(\mathcal{G} ; \mathbb{X})$ to be all $\mathbb{X}$-valued, $\mathcal{G}$-measurable random variable $\xi$ such that $\|\xi\|_{2}^{2}:=$ $\mathbb{E}\left[|\xi|^{2}\right]<\infty$. The inner product in $\mathbb{L}^{2}(\mathcal{G} ; \mathbb{X})$ is denoted by $(\xi, \eta)_{2}:=\mathbb{E}[(\xi, \eta)]$, $\xi, \eta \in \mathbb{L}^{2}(\mathcal{G} ; \mathbb{X})$.

- $\mathbb{L}_{\mathbb{F}}^{2}([s, t] ; \mathbb{X})$ to be all $\mathbb{X}$-valued, $\mathbb{F}$-adapted process $\eta$ on $[s, t]$, such that

$$
\|\eta\|_{2, s, t}:=\mathbb{E}\left[\int_{s}^{t}\left|\eta_{t}\right|^{2} d t\right]^{1 / 2}<\infty .
$$

In particular, if $\mathbb{X}=\mathbb{R}$, we shall omit $\mathbb{X}$ in the above notation for simplicity.

In what follows, we present several examples of time-inconsistent optimization problems. In each of these examples, we shall see the BSDE formulation of the original problem and the possibility of finding the dynamic utility. For simplicity, in this section we assume $d=1$.

2.1. A mean-variance optimization problem. Consider a simple controlled stochastic dynamics

$$
\begin{aligned}
X_{s}^{u}= & x_{0}+\int_{0}^{s} u_{r} d r+\int_{0}^{s} u_{r} d B_{r}, \\
& s \in[0, T], u \in \mathscr{U}_{[0, T]}:=L_{\mathbb{F}}^{2}([0, T]) .
\end{aligned}
$$

Let $c>0$ be a constant, and consider the optimization problem

$$
V_{0}:=\sup _{u \in \mathscr{U}[0, T]}\left\{\mathbb{E}\left[X_{T}^{u}\right]-\frac{1}{2 c} \operatorname{Var}\left(X_{T}^{u}\right)\right\} .
$$

Following the arguments in [20], one shows that the above optimization problem has an optimal feedback control: $u^{*}(s, x)=x_{0}-x+c e^{T}, 0 \leq s \leq T$. In other words, the optimal control is: $u_{s}^{*}=u^{*}\left(s, X^{*}\right)=x_{0}-X_{s}^{*}+c e^{T}, s \in[0, T]$, where $X^{*}$ is the corresponding optimal dynamics satisfying

$$
X_{s}^{*}=x_{0}+\int_{0}^{s}\left[x_{0}-X_{r}^{*}+c e^{T}\right] d r+\int_{0}^{s}\left[x_{0}-X_{r}^{*}+c e^{T}\right] d B_{r}, \quad s \in[0, T] .
$$

Now let $0<t<T$ be given, and we follow the control $u^{*}$ on $[0, t]$ so that $X_{t}^{*}$ is well defined. Consider the optimization problem on $[t, T]$, starting from $X_{t}^{*}$ :

$$
X_{s}^{t, u}=X_{t}^{*}+\int_{t}^{s} u_{r} d r+\int_{t}^{s} u_{r} d B_{r}, \quad s \in[t, T]
$$


and define, similar to (2.2), the value of the optimization problem at time $t$ :

$$
V_{t}:=\operatorname{esssup}_{u \in \mathscr{U}_{[t, T]}}\left\{\mathbb{E}_{t}\left[X_{T}^{t, u}\right]-\frac{1}{2 c} \operatorname{Var}_{t}\left(X_{T}^{t, u}\right)\right\},
$$

where $\operatorname{Var}_{t}$ is the conditional variance under $\mathbb{E}_{t}$. Again, as before we should have optimal control on $[t, T]: u^{t, *}(s, x)=X_{t}^{*}-x+c e^{T-t}, s \in[t, T]$. It is clear that $u^{t, *}(s, x) \neq u^{*}(s, x)$. Consequently, $u_{s}^{t, *}:=u^{t, *}\left(s, X_{s}^{t, *}\right) \neq u_{s}^{*}$, where

$$
\begin{aligned}
X_{s}^{t, *}= & X_{t}^{*}+\int_{t}^{s}\left[X_{t}^{*}-X_{r}^{t, *}+c e^{T-t}\right] d r \\
& +\int_{t}^{s}\left[X_{t}^{*}-X_{r}^{t, *}+c e^{T-t}\right] d B_{r}, \quad s \in[t, T] .
\end{aligned}
$$

Thus, the problem (2.3)-(2.4) is time inconsistent.

However, we should note that we can change the cost functional in (2.4) slightly so that it becomes time consistent. In fact, let $c_{t}>0$ be a random process and consider

$$
\tilde{V}_{t}:=\underset{u \in \mathscr{U}[t, T]}{\operatorname{esssup}}\left\{\mathbb{E}_{t}\left[X_{T}^{t, u}\right]-\frac{1}{2 c_{t}} \operatorname{Var}_{t}\left(X_{T}^{t, u}\right)\right\} .
$$

A similar argument would lead us to the optimal feedback control: $\tilde{u}^{t, *}(s, x)=$ $X_{t}^{*}-x+c_{t} e^{T-t}$. If we set

$$
c_{t}:=c e^{t}-e^{t-T}\left[X_{t}^{*}-x_{0}\right], \quad t \in[0, T],
$$

then $\tilde{u}^{t, *}(s, x)=x_{0}-x+c e^{T}=u^{*}(s, x)$. Namely, the problem (2.3), (2.5) is time consistent.

REMARK 2.1. (i) Since $c_{0}=c$, we have $\tilde{V}_{0}=V_{0}$. To wit, $\left\{\tilde{V}_{t}\right\}_{0 \leq t \leq T}$ is a timeconsistent dynamic system with initial value $V_{0}$, as desired.

(ii) We note that in the portfolio selection problems, the constant $c$ in (2.2) usually stands for the risk aversion parameter of the investor. In practice, it is reasonable that this risk aversion parameter may evolve as time changes. A timeinconsistent problem where the constant $c$ depends on state process $X$ was studied in [3]. Our example shows that if $c_{t}$ is chosen correctly, then the problem could become time consistent.

(iii) A discrete case in the same spirit of this example was studied in [8].

It is worth noting that the parameter $c_{t}$ in (2.6) is constructed via the optimal control $u^{*}$ (and so will be the examples in Sections 2.2, 2.3), which is undesirable given our goal of tackling the time inconsistency without using optimal strategy. Such a slight drawback notwithstanding, an important observation from this example is that the problems (2.1)-(2.2) can be converted to an optimal control problem 
for a 2-dimensional backward SDE:

$$
\begin{aligned}
V_{0} & :=\sup _{u \in \mathscr{U}} \varphi\left(Y_{0}^{1, u}, Y_{0}^{2, u}\right) \quad \text { where } \varphi\left(y_{1}, y_{2}\right):=y_{1}+\frac{1}{2 c}\left|y_{1}\right|^{2}-\frac{1}{2 c} y_{2}, \\
\text { (2.7) } Y_{t}^{1, u} & =X_{T}^{u}-\int_{t}^{T} Z_{s}^{1, u} d B_{s}, \\
Y_{t}^{2, u} & =\left|X_{T}^{u}\right|^{2}-\int_{t}^{T} Z_{s}^{2, u} d B_{s}, \quad t \in[0, T] .
\end{aligned}
$$

As we pointed out in the Introduction and will articulate more in the next section, one of the main reasons for the time inconsistency is the lack of comparison principle for the underlying dynamics, which is particularly the case for (2.7).

2.2. A one-dimensional example. Besides the comparison principle as mentioned in the end of the previous subsection, another reason for time inconsistency is that the $\varphi$ in (2.7) is not monotone. In what follows, we present a onedimensional example where the comparison principle holds true.

Let $\mathscr{U}:=L_{\mathbb{F}}^{2}([0, T] ;[-1,1])$. Consider a simple one-dimensional BSDE

$$
Y_{s}^{u}=B_{T}+\int_{s}^{T} u_{r} d r-\int_{s}^{T} Z_{r}^{u} d B_{r}, \quad s \in[0, T], u \in \mathscr{U},
$$

and let $\varphi(y):=-|c+y|, y \in \mathbb{R}$, for some constant $c \in \mathbb{R}$. We define the optimal value by

$$
V_{0}:=\sup _{u \in \mathscr{U}} \varphi\left(Y_{0}^{u}\right)=\sup _{u \in \mathscr{U}} \varphi\left(\mathbb{E}\left[Y_{0}^{u}\right]\right)=-\inf _{u \in \mathscr{U}}\left|c+\int_{0}^{T} \mathbb{E}\left[u_{s}\right] d s\right| .
$$

Then one can easily check that $u^{*} \in \mathscr{U}$ is an optimal control if and only if

$$
\begin{array}{rlrl}
u_{s}^{*} & \equiv-1 & \text { if } c \geq T ; \\
u_{s}^{*} \equiv 1 & \text { if } c \leq-T ; \quad \text { and } \\
\int_{0}^{T} \mathbb{E}\left[u_{s}\right] d s & =-c \quad \text { if }|c|<T .
\end{array}
$$

Now assume $c=T$. Let $0<t<T$ and consider the optimization problem over $[t, T]$ :

$$
V_{t}:=\underset{u \in \mathscr{U}}{\operatorname{esssup}} \varphi\left(Y_{t}^{u}\right)=-\underset{u \in \mathscr{U}}{\operatorname{essinf}}\left|T+B_{t}+\int_{t}^{T} \mathbb{E}_{t}\left[u_{s}\right] d s\right| .
$$

Since $c=T$, if the problem were time consistent we would then expect that the optimal control is $u_{s}^{*}=-1$, from the previous argument. However, we note that on the set $\left\{B_{t} \leq t-2 T\right\}$, one has

$$
0 \geq T+B_{t}+(T-t) \geq T+B_{t}+\int_{t}^{T} \mathbb{E}_{t}\left[u_{s}\right] d s \quad \text { for all } u \in \mathscr{U},
$$


thus the optimal control for $V_{t}$ should be $u_{s}^{t, *}=1$ on the set $\left\{B_{t} \leq t-2 T\right\}$, instead of $u_{s}^{*}=-1$, a contradiction. Namely, the problem (2.9) is time inconsistent.

Similar to the example in the previous subsection, if we allow the constant $c$ in (2.9) to be time varying and even random, then the problem could become time consistent. Indeed, if we choose $c_{t}:=T-t-B_{t}$, and consider

$$
\tilde{V}_{t}:=\underset{u \in \mathscr{U}}{\operatorname{esssup}} \Phi\left(t, Y_{t}^{u}\right) \quad \text { where } \Phi(t, y):=-\left|c_{t}+y\right| \text {. }
$$

Then it is readily seen that

$$
\begin{aligned}
\tilde{V}_{t} & =-\underset{u \in \mathscr{U}}{\operatorname{essinf}}\left|\left(T-t-B_{t}\right)+B_{t}+\int_{t}^{T} \mathbb{E}_{t}\left[u_{s}\right] d s\right| \\
& =-\underset{u \in \mathscr{U}}{\operatorname{essinf}}\left|T-t+\int_{t}^{T} \mathbb{E}_{t}\left[u_{s}\right] d s\right|,
\end{aligned}
$$

and thus the optimal control is still $u^{*}=-1$.

2.3. A principal-agent problem. In this example, we consider a special case of the Holmstrom-Milgrom model in the Pringcipal-agent Problem (cf. [9]). In this problem, the principal is to find the optimal contract assuming the agent(s) will always perform optimally given any contract. The main feature of principal's contract is that it is pre-committed, that is, it cannot be changed (at least not frequently) during a contractually designed duration.

To be more precise, let $\gamma_{A}>0, \gamma_{P}>0, R<0$ be constants, and consider two exponential utility functions:

$$
U_{A}(x):=-\exp \left\{-\gamma_{A} x\right\}, \quad U_{P}(x):=-\exp \left\{-\gamma_{P} x\right\} .
$$

We denote the principal's control set by $\mathscr{U}_{P} \subset \mathbb{L}^{2}\left(\mathcal{F}_{T}\right)$, and the agent's control set by $\mathscr{U}_{A} \subset \mathbb{L}_{\mathbb{F}}^{2}([0, T])$, satisfying certain technical conditions which for simplicity we will not specify. Given any contract $C_{T} \in \mathscr{U}_{P}$ at $t=0$, we consider the agent's problem:

$$
V_{0}^{A}\left(C_{T}\right):=\sup _{u \in \mathscr{U}_{A}} \mathbb{E}^{\mathbb{P}^{u}}\left[U_{A}\left(C_{T}-\frac{1}{2} \int_{0}^{T}\left|u_{s}\right|^{2} d s\right)\right],
$$

where $\mathbb{P}^{u}$ is a new probability measure defined by $\frac{d \mathbb{P}^{u}}{d \mathbb{P}_{0}}:=\exp \left\{\int_{0}^{T} u_{s} d B_{s}-\right.$ $\left.\frac{1}{2} \int_{0}^{T}\left|u_{s}\right|^{2} d s\right\}$. We note that here the agent's control problem (2.12) is in a "weak formulation," and $V_{0}^{A}\left(C_{T}\right) \leq 0$ is well defined. We shall consider those contracts that satisfy the following "participation constraint":

$$
V_{0}^{A}\left(C_{T}\right) \geq R
$$

where $R<0$ is the "market value" of an agent that a principal has to consider at $t=0$. 
It can be shown (cf. [9], Chapter 6) that the agent's problem can be solved in terms of the following quadratic BSDE:

$$
Y_{s}^{A}=C_{T}-\frac{\gamma_{A}-1}{2} \int_{s}^{T}\left|Z_{r}^{A}\right|^{2} d r-\int_{s}^{T} Z_{r}^{A} d B_{r}, \quad s \in[0, T] .
$$

In fact, by a simple comparison argument for BSDEs one shows that the agent's optimal action is $u^{*}=u^{*}\left(C_{T}\right)=Z^{A} \in \mathscr{U}_{A}$, with optimal value $V_{0}^{A}=U_{A}\left(Y_{0}^{A}\right)$.

Given the optimal $u^{*}=u^{*}\left(C_{T}\right)$, we now consider the principal's problem:

$$
V_{0}^{P}:=\sup _{C_{T} \in \mathscr{U}_{P}} \mathbb{E}^{\mathbb{P}^{u^{*}}}\left[U_{P}\left(B_{T}-C_{T}\right)\right]
$$

subject to the participation constraint (2.13). The solution to the problem (2.14)(2.13) can be found explicitly (cf. [9], Chapter 6). Indeed, the optimal contract is

$$
C_{T}^{*}:=-\frac{1}{\gamma_{A}} \ln (-R)+u^{*} B_{T}+\frac{\gamma_{A}-1}{2}\left|u^{*}\right|^{2} T,
$$

where $u^{*}:=\frac{1+\gamma_{P}}{1+\gamma_{A}+\gamma_{P}}$ is the corresponding agent's optimal action.

We now consider the dynamic version of the agent's problem (2.12): for $t \in$ $[0, T]$,

$$
V_{t}^{A}\left(C_{T}\right):=\underset{u \in \mathscr{U}_{A}}{\operatorname{essup}} \mathbb{E}_{t}^{\mathbb{P} u}\left[U_{A}\left(C_{T}-\frac{1}{2} \int_{t}^{T}\left|u_{s}\right|^{2} d s\right)\right],
$$

and the principal's problem, given agent's optimal control $u\left(t, C_{T}\right)$ :

$$
V_{t}^{P}:=\operatorname{Csssup}_{C_{T} \in \mathscr{U}_{P}} \mathbb{E}_{t}^{\mathbb{P}^{u\left(t, C_{T}\right)}}\left[U_{p}\left(B_{T}-C_{T}\right)\right] \quad \text { subject to } V_{t}^{A}\left(C_{T}\right) \geq R .
$$

Solving the principal's problem (2.16) as before, we see that the optimal contract is

$$
C_{T}^{t, *}:=-\frac{1}{\gamma_{A}} \ln (-R)+u^{*}\left(B_{T}-B_{t}\right)+\frac{\gamma_{A}-1}{2}\left|u^{*}\right|^{2}(T-t) .
$$

Clearly, $C_{T}^{t, *}$ is different from $C_{T}^{*}$, thus the problem is time inconsistent.

Again, the time inconsistency can be removed if we allow the market value of the agents, the constant $R$, to be time varying (as it should be). Indeed, if we set

$$
R_{t}:=R \exp \left(-\gamma_{A}\left[u^{*} B_{t}+\frac{\gamma_{A}-1}{2}\left|u^{*}\right|^{2} t\right]\right),
$$

and modify the participation constraint of the principal's problem in (2.14) to $V_{t}^{A}\left(C_{T}\right) \geq R_{t}$. Then the optimal solution to the principal's problem (2.16) will become

$$
\begin{aligned}
\tilde{C}_{T}^{t, *} & =-\frac{1}{\gamma_{A}} \ln \left(-R_{t}\right)+u^{*}\left(B_{T}-B_{t}\right)+\frac{\gamma_{A}-1}{2}\left|u^{*}\right|^{2}(T-t) \\
& =-\frac{1}{\gamma_{A}} \ln (-R)+u^{*} B_{T}+\frac{\gamma_{A}-1}{2}\left|u^{*}\right|^{2} T=C_{T}^{*},
\end{aligned}
$$


that is, the problem becomes time consistent.

We note that the problem (2.14) can also be written as an optimal control problem for a forward-backward SDE. To see this, we first note that by some straightforward arguments, one can show that for the optimal contract $C_{T}^{*}$, the identity $V_{0}\left(C_{T}^{*}\right)=R$ must hold. Therefore, we may impose a stronger participation constraint in (2.14): $V_{0}\left(C_{T}\right)=R$, and rewrite $Y^{A}$ as a forward diffusion:

$$
Y_{s}^{A}=U_{A}^{-1}(R)+\frac{\gamma_{A}-1}{2} \int_{0}^{s}\left|Z_{r}^{A}\right|^{2} d r+\int_{0}^{s} Z_{r}^{A} d B_{r}, \quad s \in[0, T],
$$

which can be thought of as the optimal solution to the agent's problem (2.14) with dynamics

$$
Y_{s}^{A, u}:=U_{A}^{-1}(R)+\frac{\gamma_{A}-1}{2} \int_{0}^{s}\left|u_{r}\right|^{2} d r+\int_{0}^{s} u_{r} d B_{r}, \quad s \in[0, T],
$$

with the relation $C_{T}=Y_{T}^{A, u}$. Then, instead of viewing $C_{T}$ as the principal's control, we may view $u:=Z^{A}$ as the principal's control, and unify the principal-agent problem to the following optimization problem for FBSDEs:

$$
V_{0}:=\sup _{u \in \mathscr{U}_{A}} Y_{0}^{P, u}
$$

where $\left(Y^{A, u}, Y^{P, u}\right)$ is the solution to the (forward) SDE (2.18) and the following BSDE:

$$
Y_{s}^{P, u}=U_{P}\left(B_{T}-Y_{T}^{A, u}\right)+\int_{s}^{T} u_{r} Z_{r}^{P, u} d r-\int_{s}^{T} Z_{r}^{P, u} d B_{r}, \quad s \in[0, T],
$$

respectively.

REMARK 2.2. The BSDEs appeared in this problem are all one-dimensional, thus the comparison principle should hold and the problem is expected to be time consistent. The time inconsistency is caused by the fixed constraint $R=V_{0}\left(C_{T}\right)$. We removed the time inconsistency by setting $R_{t}=V_{t}^{A}\left(C_{T}^{*}\right)$ for all $t \in[0, T]$, where $C_{T}^{*}=Y_{T}^{A, u^{*}}$ is the optimal contract, which is exactly the random participation constraint (2.17). In more general models, however, the BSDEs could very well be multidimensional; see, for example, [9], and the comparison principle would indeed fail.

2.4. The probability distortion problem. In this subsection, we show that the probability distortion problem considered in [33] can also be recast as an optimization problem with controlled BSDEs. With a slight variation, the problem in [33] can be understood as follows:

$$
V_{0}:=\sup _{\tau} \int_{0}^{\infty} w\left(\mathbb{P}_{0}\left(U\left(B_{\tau}\right) \geq x\right)\right) d x
$$


where $\tau$ is running over all stopping times, $U \geq 0$ is a utility function and the probability distortion function $w:[0,1] \rightarrow[0,1]$ is a continuous and strictly increasing function such that $w(0)=0$ and $w(1)=1$. If $w(x)=x$ for all $x \in[0,1]$, then $V_{0}=\sup _{\tau} \mathbb{E}\left[U\left(B_{\tau}\right)\right]$, which is a standard optimal stopping problem and is time consistent. However, for general distortion function $w$, the problem is typically time inconsistent as was showed in [33], where the optimal stopping time was constructed by using some quantile functions and the Skorohod embedding theorem.

To write (2.21) in the form of (1.5), we let $\tau$ be the control and $x \in[0, \infty)$ be the parameter. For each $x$ and $\tau$, introduce a BSDE:

$$
Y_{t}^{x, \tau}=\mathbf{1}_{\left\{U\left(B_{\tau}\right) \geq x\right\}}-\int_{t}^{T} Z_{s}^{x, \tau} d B_{S} .
$$

That is, we view $Y^{\tau}:=\left(Y^{x, \tau}\right)_{x \in[0, \infty)}$ as the solution to a (uncountably) infinite dimensional BSDE. Then we have

$$
V_{0}=\sup _{\tau} \varphi\left(Y_{0}^{\tau}\right) \quad \text { where } \varphi(f):=\int_{0}^{\infty} w(f(x)) d x .
$$

2.5. A deterministic example. It is a common suspicion that the random uncertainty involved in the underlying problem may play some fundamental role in the time inconsistency. To conclude this section, we provide a simple deterministic example where the comparison principle fails in order to show that the time inconsistency is more of a structural issue than an information issue.

Let $T>1$, and $\mathscr{U}_{[s, t]}$ be the set of deterministic functions $u:[s, t] \rightarrow[0,1]$. Consider the deterministic optimization problem:

$$
\begin{aligned}
V_{t}:= & \sup _{u \in \mathscr{U}[t, T]} Y_{t}^{1, u}, \\
& \text { where } Y_{t}^{1, u}:=\int_{t}^{T}\left[u_{s}-Y_{s}^{2, u}\right] d s, Y_{t}^{2, u}:=\int_{t}^{T} u_{s} d s, t \in[0, T] .
\end{aligned}
$$

By straightforward calculation, we obtain that

$$
Y_{t}^{1, u}=\int_{t}^{T}\left[u_{s}-\int_{s}^{T} u_{r} d r\right] d s=\int_{t}^{T}[1+t-s] u_{s} d s
$$

and then clearly the optimal control is: $u_{s}^{t, *}:=\mathbf{1}_{[t,(1+t) \wedge T]}(s), t \leq s \leq T$. In particular, for $0<t<T-1$, we see that

$$
u_{s}^{0, *}=0 \neq 1=u_{s}^{t, *}, \quad s \in(1,1+t) .
$$

That is, the problem (2.24) is time inconsistent. 
3. Characterization of time consistency in our model. Having argued in previous section that many time-inconsistent problems can be recasted as optimization problems with controlled BSDEs/FBSDEs, in the rest of the paper we shall focus exclusively on such class of optimization problems and introduce our main schemes. Again, our purpose here is to revitalize the "dynamical programming principle" (DPP) in a time-inconsistent situation, without assuming the existence of an optimal control. As we pointed out in Introduction, in order to focus more on the main ideas, we shall consider only the case where the controlled dynamics are finite dimensional BSDEs, with the forward component being simply the driving Brownian motion itself. The extension to controlled forward SDEs requires some heavier notation but no substantial difficulty. The generalization to infinite dimension is more challenging in general, and we shall leave it to future study.

We begin with a precise description of the framework. Let $U$ be a Polish set, and $\mathscr{U}:=\mathbb{L}_{\mathbb{F}}^{0}([0, T] ; U)$. Consider the following $d^{\prime}$-dimensional BSDE:

$$
Y_{t}^{u}=\xi+\int_{t}^{T} f\left(s, Y_{s}^{u}, Z_{s}^{u}, u_{s}\right) d s-\int_{t}^{T} Z_{s}^{u} d B_{s}, \quad t \in[0, T] .
$$

Now, for a given cost function $\varphi: \mathbb{R}^{d^{\prime}} \rightarrow \mathbb{R}$, we define the following optimization problem:

$$
V_{0}(\xi):=\sup _{u \in \mathscr{U}} \varphi\left(Y_{0}^{u}\right), \quad \text { for any } \xi \in \mathbb{L}^{2}\left(\mathcal{F}_{T} ; \mathbb{R}^{d^{\prime}}\right) .
$$

Throughout this paper, we shall make use of the following standing assumptions.

Assumption 3.1. (i) The generator $f:[0, T] \times \Omega \times \mathbb{R}^{d^{\prime}} \times \mathbb{R}^{d^{\prime} \times d} \times U \rightarrow \mathbb{R}^{d^{\prime}}$ is $\mathbb{F}$-progressively measurable in all variables, uniformly Lipschitz continuous in $(y, z)$, and

$$
\mathbb{E}\left[\left(\int_{0}^{T} \sup _{u \in U}|f(t, 0,0, u)| d t\right)^{2}\right]<\infty .
$$

(ii) The function $\varphi: \mathbb{R}^{d^{\prime}} \rightarrow \mathbb{R}$ is continuous.

Given $\xi \in \mathbb{L}^{2}\left(\mathcal{F}_{T} ; \mathbb{R}^{d^{\prime}}\right)$, it is by now well understood that, under Assumption 3.1, BSDE (3.1) is well-posed for any $u \in \mathscr{U}$, and $\left\{Y_{0}^{u}, u \in \mathscr{U}\right\}$ is a bounded set in $\mathbb{R}^{d^{\prime}}$. Thus, $V_{0}(\xi)$ in (3.2) is well defined. We shall refer to problem (3.2) as the static problem.

We now consider the problem (3.2) in a dynamic setting. For $0 \leq t \leq T$, we define

$$
V_{t}(\xi):=\underset{u \in \mathscr{U}}{\operatorname{esssup}} \varphi\left(Y_{t}^{u}\right)
$$


As we observed in the previous section, when $\varphi$ is nonmonotone or when $d^{\prime} \geq 2$, the problem (3.3) is typically time inconsistent in the sense that the optimal control of static problem (3.2) is no longer optimal for the dynamic problem (3.3) over the time duration $[t, T]$. We should note, however, that such a characterization, although self-explanatory and easy to understand, has a fundamental drawback, that is, it relies on the existence of optimal control, which in general is a tall order. In fact, it is by no means clear why problems (3.2) and (3.3) will possess any optimal control, which in theory would make it impossible to check the time consistency of the problem.

To get around this deficiency, we propose a more generic characterization of time inconsistency, based on the DPP for the value function. To facilitate our discussion, let us introduce another notation. For any $0<t \leq T, \eta \in \mathbb{L}^{2}\left(\mathcal{F}_{t}\right)$, and $u \in \mathscr{U}$, let $\left(\mathscr{Y}^{u}(t, \eta), \mathscr{Z}^{u}(t, \eta)\right)$ denote the solution to the following BSDE on $[0, t]$ :

$$
\mathscr{Y}_{s}^{u}=\eta+\int_{s}^{t} f\left(r, \mathscr{Y}_{r}^{u}, \mathscr{Z}_{r}^{u}, u_{r}\right) d r-\int_{s}^{t} \mathscr{Z}_{r}^{u} d B_{r}, \quad 0 \leq s \leq t .
$$

Clearly, using the notation $\mathscr{Y}^{u}(\cdot, \cdot)$ and uniqueness of the solution to BSDE (3.4) we can write: $Y_{s}^{u}=\mathscr{Y}_{s}^{u}\left(t, Y_{t}^{u}\right), 0 \leq s \leq t \leq T$; and, in particular, $Y_{0}^{u}=\mathscr{Y}_{0}^{u}\left(t, Y_{t}^{u}\right)$, $t \in[0, T]$.

We illustrate the idea through two examples where $\varphi$ is monotone and the BSDE satisfies the comparison principle.

EXAMPLE 3.2. Assume that Assumption 3.1 is in force, and assume further that $d^{\prime}=1$ and $\varphi$ is increasing. Then it is clear that the static problem (3.2) is equivalent to $V_{0}(\xi):=\varphi\left(\sup _{u \in \mathscr{U}} Y_{0}^{u}\right)$. On the other hand, by the comparison principle of BSDEs and the monotonicity of $\varphi$, we see immediately that the dynamic problem (3.3) can also be written as $V_{t}(\xi)=\varphi\left(\bar{Y}_{t}\right), 0 \leq t \leq T$, where $\bar{f}(s, \omega, y, z):=\sup _{u \in U} f(s, \omega, y, z, u)$, and

$$
\bar{Y}_{s}=\xi+\int_{s}^{T} \bar{f}\left(r, \bar{Y}_{r}, \bar{Z}_{r}\right) d r-\int_{s}^{T} \bar{Z}_{r} d B_{r}, \quad s \in[0, T] .
$$

We claim that this problem is time consistent in the sense that the following DPP holds:

$$
V_{t_{1}}(\xi)=\operatorname{esssup}_{u \in \mathscr{U}} \varphi\left(\mathcal{Y}_{t_{1}}^{u}\left(t_{2}, \bar{Y}_{t_{2}}\right)\right), \quad \mathbb{P}_{0} \text {-a.s., } 0 \leq t_{1}<t_{2} \leq T .
$$

Indeed, for simplicity we set $t_{1}:=0$ and $t_{2}:=t$. For any $u \in \mathscr{U}$, we write $Y_{0}^{u}=$ $\mathcal{Y}_{0}^{u}\left(t, Y_{t}^{u}\right)$. By the comparison principle of BSDE, we see that $Y_{t}^{u} \leq \bar{Y}_{t}$, which implies $Y_{0}^{u}=\mathscr{Y}_{0}^{u}\left(t, Y_{t}^{u}\right) \leq \mathscr{Y}_{0}^{u}\left(t, \bar{Y}_{t}\right)$, and consequently $\varphi\left(Y_{0}^{u}\right) \leq \varphi\left(\mathscr{Y}_{0}^{u}\left(t, \bar{Y}_{t}\right)\right)$, thanks to the monotonicity of $\varphi$. Since $u$ is arbitrary, we conclude that

$$
V_{0}(\xi) \leq \sup _{u \in \mathscr{U}} \varphi\left(\mathscr{Y}_{0}^{u}\left(t, \bar{Y}_{t}\right)\right)
$$


To see the opposite inequality of (3.6), for any $\varepsilon>0$, we apply the standard measurable selection theorem to get a measurable function $I_{\varepsilon}:[0, T] \times \Omega \times \mathbb{R} \times$ $\mathbb{R}^{1 \times d} \rightarrow U$ such that

$$
f\left(s, \omega, y, z, I_{\varepsilon}(s, \omega, y, z)\right) \geq \bar{f}(s, \omega, y, z)-\varepsilon, \quad \forall(s, \omega, y, z) .
$$

Set $u_{s}^{\varepsilon}:=I_{\varepsilon}\left(s, \bar{Y}_{s}, \bar{Z}_{s}\right), t \leq s \leq T$. By standard BSDE arguments, we see that

$$
\bar{Y}_{t} \leq Y_{t}^{u^{\varepsilon}}+C \varepsilon .
$$

Now for any $u \in \mathscr{U}$, by standard BSDE arguments again, it follows from (3.8) that

$$
\mathscr{Y}_{0}^{u}\left(t, \bar{Y}_{t}\right) \leq \mathscr{Y}_{0}^{u}\left(t, Y_{t}^{u^{\varepsilon}}\right)+C \varepsilon=Y_{0}^{u \otimes_{t} u^{\varepsilon}}+C \varepsilon \leq V_{0}(\xi)+C \varepsilon,
$$

where $u \bigotimes_{t} u^{\varepsilon}:=u \mathbf{1}_{[0, t)}+u^{\varepsilon} \mathbf{1}_{[t, T]}$. By the arbitrariness of $u$ and $\varepsilon$, we prove the opposite inequality in (3.6), whence the DPP (3.5).

We should note that the DPP (3.5) does not require the existence of optimal control, but it indeed characterizes the time consistency. Moreover, when $U$ is compact and $f$ is continuous in $u$, there exists a measurable function $I:[0, T] \times$ $\Omega \times \mathbb{R} \times \mathbb{R}^{1 \times d} \rightarrow U$ such that

$$
f(s, \omega, y, z, I(s, \omega, y, z))=\bar{f}(s, \omega, y, z), \quad \forall(s, \omega, y, z) .
$$

In this case, one can easily check that $u_{s}^{*}:=I\left(s, \bar{Y}_{s}, \bar{Z}_{s}\right)$ is optimal both for $V_{0}(\xi)$ and for any $V_{t}(\xi)$. So the problem is time consistent in terms of optimal control as well.

REMARK 3.3. As we see in the argument leading to (3.6), the DPP (3.5) clearly relies on both the comparison principle of the BSDE and the monotonicity of $\varphi$. In fact, as we saw in Section 2.2, the comparison principle alone is not sufficient for the time consistency.

The next example reinforces the importance of comparison principle for time consistency.

EXAMPLE 3.4. Let $d^{\prime} \geq 2$. Consider the following multidimensional BSDE: for $i=1, \ldots, d^{\prime}$,

$$
\bar{Y}_{t}^{i}=\xi_{i}+\int_{t}^{T} \bar{f}_{i}\left(s, \bar{Y}_{s}, \bar{Z}_{s}^{i}\right) d s-\int_{t}^{T} \bar{Z}_{s}^{i} d B_{s},
$$

where $\bar{f}_{i}\left(t, y, z_{i}\right):=\sup _{u \in U} f_{i}\left(t, y, z_{i}, u\right)$. Assume that:

(i) for $i=1, \ldots, d^{\prime}, f_{i}$ does not depend on $z_{j}$ and is increasing in $y_{j}$, for all $j \neq i$; and

(ii) $\varphi$ is increasing in each component. 
Then it is well known that the comparison principle remains true for such BSDEs. Following the similar arguments as in Example 3.2, we can show that $V_{t}(\xi)=$ $\varphi\left(\bar{Y}_{t}\right), 0 \leq t \leq T$, and

$$
V_{t_{1}}(\xi)=\operatorname{esssup}_{u \in \mathscr{U}} \varphi\left(\mathscr{Y}_{t_{1}}^{u}\left(t_{2}, \bar{Y}_{t_{2}}\right)\right), \quad \mathbb{P}_{0} \text {-a.s., } \quad 0 \leq t_{1}<t_{2} \leq T .
$$

Consequently, the problem is time consistent.

From Example 3.4, we see the crucial roles that the comparison principle and the monotonicity of some key coefficients play in the time consistency. In general, the comparison principle fails for $d^{\prime}>2$ except for some special cases. We refer to [21] for some detailed analysis on this issue. We note that the problem will remain time consistent if $f_{i}$ and $\varphi$ are monotone on the corresponding variables in a compatible manner (e.g., $f_{i}$ is decreasing in $y_{j}$ and $\varphi$ is decreasing in all its variables). The result would be very different if such compatibility is violated. In fact, as we saw in Section 2.5, when $f_{i}$ is decreasing in $y_{j}$ but $\varphi$ is increasing, the problem becomes time inconsistent.

To study the general time-inconsistent problem, we propose the following definition.

DEFINITION 3.5. An F-progressively measurable function $\Phi:[0, T] \times \Omega \times$ $\mathbb{R}^{d^{\prime}} \rightarrow \mathbb{R}$ is called a "time-consistent dynamic utility function" for problems (3.1)(3.2) if:

(i) $\Phi(0, y)=\varphi(y)$,

(ii) there exists a mapping $\bar{Y}:[0, T] \times \Omega \mapsto \mathbb{R}^{d^{\prime}}$ satisfying $\bar{Y}_{t} \in \mathbb{L}^{2}\left(\mathcal{F}_{t} ; \mathbb{R}^{d^{\prime}}\right)$, for $t \in[0, T]$ and $\bar{Y}_{T}=\xi, \mathbb{P}$-a.s., such that the following DPP holds:

$$
\Phi\left(t_{1}, \bar{Y}_{t_{1}}\right)=\operatorname{esssup}_{u \in \mathscr{U}} \Phi\left(t_{1}, \mathscr{Y}_{t_{1}}^{u}\left(t_{2}, \bar{Y}_{t_{2}}\right)\right), \quad 0 \leq t_{1}<t_{2} \leq T .
$$

In particular, in this case we say that the following dynamic processes is time consistent:

$$
\tilde{V}_{t}(\xi):=\Phi\left(t, \bar{Y}_{t}\right)=\underset{u \in \mathscr{U}}{\operatorname{esssup}} \Phi\left(t, Y_{t}^{u}\right)
$$

REMARK 3.6. The time-consistent dynamic utility function $\Phi$ is motivated in part by the notion of the forward utility proposed in [17, 28, 29], because both evolve forwardly in time. It should be noted, however, that there is a fundamental difference here: for each $t \in[0, T]$, the forward utility $U(t, \cdot)$ in $[17,28,29]$ acts on $t$ and optimizes over the time duration $[0, t]$, whereas our dynamic utility $\Phi(t, \cdot)$ acts on terminal time $T$ and optimizes over the time duration $[t, T]$.

We would like to emphasize the following three main features of Definition 3.5: 
(1) $\tilde{V}_{0}(\xi)=V_{0}(\xi)$, thanks to condition (i). This means the dynamic problem coincides with the static problem.

(2) The function $\Phi$ is defined "forwardly," with an initial value, and the mapping $\bar{Y}$ is defined backwardly, with a terminal value. We should particularly note that at this point we do not require the $t$-measurability of the mapping $\bar{Y}$; and

(3) The time consistency is characterized by the DPP, which does not require the existence of optimal control.

It is easy to see that the function $\Phi(t, \cdot) \equiv \varphi$ in Examples 3.2 and 3.4 is a time-consistent dynamic utility. Furthermore, if the optimal control $u^{*}$ exists, we may simply set $\bar{Y}:=Y^{u^{*}}$, and in this case one can easily find a desired $\Phi$, as we see in the examples in the previous section. However, in general, we need to find the $\bar{Y}$ whose dynamics (if it exists) may help us to either determine the optimal control $u^{*}$, if any, or find conditions for the existence of optimal control. We should also note that the dynamic utility function $\Phi$ is not unique. In fact, if $\Phi$ is a time-consistent dynamic utility, then for any process $\theta$ with $\theta_{0}=0$, $\tilde{\Phi}(t, y):=\Phi(t, y)+\theta_{t}$ is also a time-consistent dynamic utility. Since our main difficulty is the existence of such $\Phi$, in Definition 3.5 we impose minimum requirements on $\Phi$.

In the rest of this paper, we shall propose three possible approaches to attack the general time-inconsistent optimization problems [in the sense that $\Phi(t, \cdot) \equiv \varphi$ is not a time-consistent dynamic utility function]. Each approach has its pros and cons. We note that in this paper we focus mainly on the ideas, rather than the actual solvability of the resulting problems, which could be highly technical, and may call for some new developments in the respective areas.

\section{The duality approach.}

4.1. Heuristic analysis in Markovian case. In this section, we present a duality approach that is simple but quite effective if one focuses only on finding the value of the static problem (3.2). To illustrate the idea better, we begin by considering the Markovian case, that is, we assume that in $\operatorname{BSDE}(3.1) \xi=g\left(B_{T}\right)$ and $f=$ $f\left(t, B_{t}, y, z, u\right)$. We shall start with heuristic arguments, and give the proof for the general non-Markovian (or say path-dependent) case.

To begin with, for each $(t, x) \in[0, T] \times \mathbb{R}^{d}$, consider the set

$$
\begin{aligned}
\mathscr{D}(t, x):= & \left\{y \in \mathbb{R}^{d^{\prime}}: \exists Z \in \mathbb{L}_{\mathbb{F}}^{2}([0, T]),\right. \\
& \left.u \in \mathscr{U}_{[t, T]}, \text { s.t. } X_{T}^{t, x, y, Z, u}=g\left(B_{T}^{t, x}\right), \mathbb{P}_{0} \text {-a.s. }\right\},
\end{aligned}
$$

where $B_{s}^{t, x}:=x+B_{s}-B_{t}, s \geq t$, and $X^{t, x, y, Z, u}$ is the solution to the forward SDE

$$
X_{s}=y-\int_{t}^{s} f\left(r, B_{r}^{t, x}, X_{r}, Z_{r}, u_{r}\right) d r+\int_{t}^{s} Z_{r} d B_{r}, \quad t \leq s \leq T .
$$


Clearly, $X$ can be thought of as a forward version of the solution to the BSDE (3.1) on $[t, T]$, and the set $\mathscr{D}(t, x)$ is simply the reachable set $\left\{Y_{t}^{u}, u \in \mathscr{U}\right\}$ given $B_{t}=x$. In particular, $\mathscr{D}(0,0)=\left\{Y_{0}^{u}: u \in \mathscr{U}\right\}$, and our original optimization (3.2) can be rewritten as

$$
V_{0}(\xi)=\sup _{y \in \mathscr{D}(0,0)} \varphi(y)
$$

It is worth noting that $\sup _{y \in \mathscr{D}(0,0)} \varphi(y)$ in (4.3) is a finite dimensional optimization problem. So the value $V_{0}(\xi)$ could be determined rather easily, provided one can characterize the set $\mathscr{D}(0,0)$, which we now describe.

To this end, we borrow the idea of the method of optimal control for solving a forward-backward SDE (cf. [26]). Consider the following dual control problem:

$$
W(t, x, y):=\inf _{Z, u} \mathbb{E}\left\{\left|X_{T}^{t, x, y, Z, u}-g\left(B_{T}^{t, x}\right)\right|^{2}\right\} .
$$

Clearly, (4.4) is a standard stochastic control problem, and it is well known that $W$ should be the (unique) viscosity solution to the following (degenerate) HJB equation:

$$
\left\{\begin{aligned}
& \partial_{t} W+\frac{1}{2} \partial_{x x}^{2} W \\
&+\inf _{z, u}\left\{\frac{1}{2} \partial_{y y}^{2} W:\left(z z^{\top}\right)+\partial_{x y}^{2} W: z-\partial_{y} W \cdot f(t, x, y, z, u)\right\}=0 \\
& W(T, x, y)=|y-g(x)|^{2}
\end{aligned}\right.
$$

By definition (4.1), it is clear that $W(t, x, y)=0$ whenever $y \in \mathscr{D}(t, x)$. More generally, we expect and will show that, for any $(t, x)$, the following duality relationship between the set $\mathscr{D}(t, x)$ and the "nodal set" of the function $W$ holds:

$$
\mathscr{N}(t, x):=\left\{y \in \mathbb{R}^{d^{\prime}}: W(t, x, y)=0\right\}=\overline{\mathscr{D}(t, x)},
$$

where $\overline{\mathscr{D}(t, x)}$ denotes the closure of $\mathscr{D}(t, x)$. Then (4.3) amounts to saying that

$$
V_{0}(\xi)=\sup _{y \in \mathscr{D}(0,0)} \varphi(y)=\sup _{y \in \mathscr{N}(0,0)} \varphi(y) \text {. }
$$

In other words, we have characterized the set $\mathscr{D}(0,0)$ in terms of $\mathscr{N}(0,0)$, the nodal set of $W$, which is a much benign task to deal with (e.g., numerically). Moreover, note that the nodal set $\mathscr{N}(0,0) \subset \mathbb{R}^{d^{\prime}}$ is closed, then the above optimization problem has a maximum argument $y^{*} \in \mathscr{N}(0,0)$. Consequently, the static optimization problem (3.2) has an optimal control if and only if there exists $y^{*} \in \mathscr{D}(0,0)$.

REMARK 4.1. (i) An important ingredient in the duality approach is the "reachable set" $\mathscr{D}(\cdot, \cdot)$. Unlike the standard optimal control literature where reachable sets are temporally forward, it is easy to see from (4.1) that the family 
$\{\mathscr{D}(t, \cdot)\}_{0 \leq t \leq T}$ is a backward, set-valued dynamic system with terminal condition $\mathscr{D}(T, x)=\{g(x)\}$, and as we shall see later in this section, it satisfies a geometric $D P P$ in the spirit of [31].

(ii) The duality approach could be combined with the time consistency in the sense of Definition 3.5 as follows. Assuming we could find a desired timeconsistent dynamic utility $\Phi$, which we hope will take the form $\Phi\left(t, B_{t}, y\right)$ in the Markovian case, then by the duality (4.6) we have the following time-consistent value function:

$$
\tilde{V}_{t}(\xi)=\operatorname{esssup}_{y \in \mathscr{N}\left(t, B_{t}\right)} \Phi\left(t, B_{t}, y\right)
$$

Moreover, since the nodal set $\mathscr{N}\left(t, B_{t}\right) \subset \mathbb{R}^{d^{\prime}}$ is closed and assuming the continuity of $\Phi$ in $y$, the above optimization problem has maximum argument $\bar{Y}_{t}$, which would serve for the purpose of Definition 3.5.

(iii) We note that the problem (4.8) will be "time consistent" in the following two senses. First, the dynamic sets $\mathscr{N}(\cdot, \cdot)$ is time consistent in the sense of a geometric DPP, which we shall establish in Theorem 4.5 below, whereas the function $\Phi$ is time consistent in the sense of Definition 3.5. Second, in the case when optimal control $u^{*}$ for the static problem (3.2) exists, and if $\Phi$ is constructed by using $u^{*}$ as we did for various examples in Section 2, then $u^{*}$ will remain optimal for the dynamic problem (4.8), and thus time consistent in the usual sense.

(iv) We should point out again that (4.8) is a simple finite dimensional optimization problem, provided that the nodal sets $\mathscr{N}$ and function $\Phi$ can be computed. In particular, when $\Phi$ is continuous in $y$, the essential supremum there is simply a supremum. However, while the set $\mathscr{N}$, as the nodal set of $W$, can be computed (at least numerically) by solving the HJB equation for $W$, the task of finding a desired $\Phi$ is generally challenging. We shall elaborate this point more in the next section.

4.2. The duality approach for the general path dependent case. We now carry out the duality approach rigorously in the general path dependent (or non-Markovian) case. To begin with, we recall the canonical set-up introduced in the beginning of Section 2. Moreover, for any $t \in[0, T]$, denote by $\Omega^{t}:=$ $\left\{\omega \in C\left([t, T], \mathbb{R}^{d}\right): \omega_{t}=0\right\}$ the shifted canonical space on $[t, T]$, and define $B^{t}, \mathbb{F}^{t}, \mathbb{P}_{0}^{t}, \Lambda^{t}, \mathscr{U}^{t}$, etc. on $\Omega^{t}$ in the obvious sense. Furthermore, for any $\omega \in \Omega$ and $\tilde{\omega} \in \Omega^{t}$, we introduce the concatenation: $\omega \bigotimes_{t} \tilde{\omega}:=\omega \mathbf{1}_{[0, t]}+\left(\omega_{t}+\tilde{\omega}\right) \mathbf{1}_{[t, T]}$. Moreover, for $\xi \in \mathbb{L}^{0}(\Omega)$ and $(t, \omega) \in \Lambda$, denote $\xi^{t, \omega}(\tilde{\omega}):=\xi\left(\omega \otimes_{t} \tilde{\omega}\right)$, for all $\tilde{\omega} \in \Omega^{t}$.

Similar to (4.1), for any $(t, \omega) \in \Lambda$ we define

$$
\begin{aligned}
\mathscr{D}(t, \omega):= & \left\{y \in \mathbb{R}^{d^{\prime}}: \exists(Z, u) \in \mathbb{L}^{2}\left(\mathbb{F}^{t}, \mathbb{R}^{d^{\prime} \times d}\right) \times \mathscr{U}^{t},\right. \\
& \text { s.t. } \left.X_{T}^{t, \omega, y, Z, u}=\xi^{t, \omega}, \mathbb{P}_{0}^{t} \text {-a.s. }\right\},
\end{aligned}
$$


where $X^{t, \omega, y, Z, u}$ is the solution to the following (forward) SDE:

$$
\begin{aligned}
X_{s}= & y-\int_{t}^{s} f^{t, \omega}\left(r, B^{t}, X_{r}, Z_{r}, u_{r}\right) d r \\
& +\int_{t}^{s} Z_{r} d B_{r}^{t}, \quad t \leq s \leq T, \mathbb{P}_{0^{-}}^{t}, \quad
\end{aligned}
$$

Here, the function $f^{t, \omega}(r, \tilde{\omega}, y, z, u),(r, \tilde{\omega}) \in \Lambda^{t}$ is defined the same as $\xi^{t, \omega}$ explained before. Again, it is easy to see that $\mathscr{D}(0,0)=\left\{Y_{0}^{u}: u \in \mathscr{U}\right\}$ remains true. Thus, we still have

$$
V_{0}(\xi)=\sup _{y \in \mathscr{D}(0,0)} \varphi(y) .
$$

We now introduce a dual control problem in the path-dependent setting:

$$
W(t, \omega, y):=\inf _{(Z, u) \in \mathbb{L}^{2}\left(\mathbb{F}^{t}, \mathbb{R}^{d^{\prime} \times d}\right) \times \mathscr{U}^{t}} \mathbb{E}^{\mathbb{P}^{t}}\left[\left|X_{T}^{t, \omega, y, Z, u}-\xi^{t, \omega}\right|^{2}\right] .
$$

Our main duality result is as follows.

THEOREM 4.2. Let Assumption 3.1 hold, and assume further that, for any $(t, \omega) \in \Lambda$,

$$
\mathbb{E}^{\mathbb{P}_{0}^{t}}\left[\left(\int_{t}^{T} \sup _{u \in U}\left|f^{t, \omega}\left(s, B_{.}^{t}, 0,0, u\right)\right| d s\right)^{2}+\left|\xi^{t, \omega}\right|^{2}\right]<\infty .
$$

Then, for any $(t, \omega) \in \Lambda$, we have

$$
\mathscr{N}(t, \omega):=\left\{y \in \mathbb{R}^{d^{\prime}}: W(t, \omega, y)=0\right\}=\overline{\mathscr{D}(t, \omega)} .
$$

Consequently, $V_{0}(\xi)=\sup _{y \in \mathscr{N}(0,0)} \varphi(y)$.

PROOF. Noting (4.11) and the continuity of $\varphi$, we shall prove only (4.14).

We first prove the regularity of $W$ in $y$ : for any $(t, \omega) \in \Lambda$, and $y_{1}, y_{2} \in \mathbb{R}$,

$$
\left|W\left(t, \omega, y_{1}\right)-W\left(t, \omega, y_{2}\right)\right| \leq C(t, \omega)\left[1+\left|y_{1}\right|+\left|y_{2}\right|\right]\left|y_{1}-y_{2}\right|,
$$

where $C(t, \omega)>0$ is independent of $y$. Indeed, by (4.12) and (4.13), it is readily seen that

$$
W(t, \omega, y) \leq C(t, \omega)\left[1+|y|^{2}\right] .
$$

Now for any $0<\varepsilon<1$, we choose $\left(Z^{\varepsilon}, u^{\varepsilon}\right) \in \mathbb{L}^{2}\left(\mathbb{F}^{t}, \mathbb{R}^{d^{\prime} \times d}\right) \times \mathscr{U}^{t}$ such that

$$
\mathbb{E}^{\mathbb{P}_{0}^{t}}\left[\left|X_{T}^{t, \omega, y_{2}, Z^{\varepsilon}, u^{\varepsilon}}-\xi^{t, \omega}\right|^{2}\right] \leq W\left(t, \omega, y_{2}\right)+\varepsilon \leq C(t, \omega)\left[1+\left|y_{2}\right|^{2}\right] .
$$

By the standard BSDE arguments, it is then clear that, under Assumptions 3.1, we have

$$
\mathbb{E}^{\mathbb{P}_{0}^{t}}\left[\left|X_{T}^{t, \omega, y_{1}, Z^{\varepsilon}, u^{\varepsilon}}-X_{T}^{t, \omega, y_{2}, Z^{\varepsilon}, u^{\varepsilon}}\right|^{2}\right] \leq C\left|y_{1}-y_{2}\right|^{2}
$$


Then, denoting $X^{i}:=X^{t, \omega, y_{i}, Z^{\varepsilon}, u^{\varepsilon}}, i=1,2$, we have

$$
\begin{aligned}
W\left(t, \omega, y_{1}\right)-W\left(t, \omega, y_{2}\right) & \leq \mathbb{E}^{\mathbb{P}_{0}^{t}}\left[\left|X_{T}^{1}-\xi^{t, \omega}\right|^{2}\right]-\mathbb{E}^{\mathbb{P}_{0}^{t}}\left[\left|X_{T}^{2}-\xi^{t, \omega}\right|^{2}\right]+\varepsilon \\
& \leq \mathbb{E}^{\mathbb{P}_{0}^{t}}\left[\left|X_{T}^{1}-X_{T}^{2}\right|^{2}+2\left|X_{T}^{1}-X_{T}^{2}\right|\left|X_{T}^{2}-\xi^{t, \omega}\right|\right]+\varepsilon \\
& \leq C\left|y_{1}-y_{2}\right|^{2}+C(t, \omega)\left[1+\left|y_{2}\right|\right]\left|y_{1}-y_{2}\right|+\varepsilon \\
& \leq C(t, \omega)\left[1+\left|y_{1}\right|+\left|y_{2}\right|\right]\left|y_{1}-y_{2}\right|+\varepsilon .
\end{aligned}
$$

Since $\varepsilon$ is arbitrary, we obtain the desired estimate (4.15) for $W\left(t, \omega, y_{1}\right)-$ $W\left(t, \omega, y_{2}\right)$. Switching the roles of $y_{1}$ and $y_{2}$, we can also obtain the estimate for $W\left(t, \omega, y_{2}\right)-W\left(t, \omega, y_{1}\right)$, whence $(4.15)$.

Next, we fix $(t, \omega) \in \Lambda$ and let $y \in \mathscr{D}(t, \omega)$. By definition, there exists $(Z, u) \in$ $\mathbb{L}^{2}\left(\mathbb{F}^{t}, \mathbb{R}^{d^{\prime} \times d}\right) \times \mathscr{U}^{t}$ such that $X_{T}^{t, \omega, y, Z, u}=\xi^{t, \omega}, \mathbb{P}_{0}^{t}$-a.s. Then we must have

$$
W(t, \omega, y) \leq \mathbb{E}^{\mathbb{P}_{0}^{t}}\left[\left|X_{T}^{t, \omega, y, Z, u}-\xi^{t, \omega}\right|^{2}\right]=0 .
$$

That is, $y \in \mathscr{N}(t, \omega)$, and consequently $\mathscr{D}(t, \omega) \subset \mathscr{N}(t, \omega)$. Moreover, the $y$ continuity of $W$ in (4.15) then implies that $\mathscr{N}(t, \omega)$ is a closed set, which leads to that $\overline{\mathscr{D}(t, \omega)} \subset \mathscr{N}(t, \omega)$.

Conversely, if $y \in \mathscr{N}(t, \omega)$, then by definition for any $\varepsilon>0$, there exists $\left(Z^{\varepsilon}, u^{\varepsilon}\right) \in \mathbb{L}^{2}\left(\mathbb{F}^{t}, \mathbb{R}^{d^{\prime} \times d}\right) \times \mathscr{U}^{t}$ such that

$$
\mathbb{E}^{\mathbb{P}_{0}^{t}}\left[\left|\xi_{\varepsilon}^{t, \omega}-\xi^{t, \omega}\right|^{2}\right] \leq \varepsilon \quad \text { where } \xi_{\varepsilon}^{t, \omega}:=X_{T}^{t, \omega, y, Z^{\varepsilon}, u^{\varepsilon}} .
$$

Now by the standard BSDE estimates we have, for the given $(t, \omega) \in \Lambda$,

$$
\left|Y_{t}^{u^{\varepsilon}}(\omega)-y\right|^{2}=\left|\mathscr{Y}_{t}^{u^{\varepsilon}}\left(T, \xi^{t, \omega}\right)-\mathscr{Y}_{t}^{u^{\varepsilon}}\left(T, \xi_{\varepsilon}^{t, \omega}\right)\right|^{2} \leq C \mathbb{E}^{\mathbb{P}_{0}^{t}}\left[\left|\xi_{\varepsilon}^{t, \omega}-\xi^{t, \omega}\right|^{2}\right] \leq C \varepsilon
$$

Since $Y_{t}^{u^{\varepsilon}}(\omega) \in \mathscr{D}(t, \omega)$ and $\varepsilon$ is arbitrary, we see that $y \in \overline{\mathscr{D}(t, \omega)}$.

4.3. Characterization of $W$ by PPDEs. It is well understood that, in the Markovian case, the dual value function $W$ is the viscosity solution to HJB equation (4.5). In this subsection, we extend this characterization of $W$ to path dependent case via the newly established viscosity theory developed in [12-14]. The path derivatives introduced here will also be important in Section 6. Since the results here are irrelevant to the rest of the paper, we shall focus only on the main ideas without getting into all the technical details. The interested readers are referred to $[13,14]$ for more on pathwise analysis involved in the arguments.

We first consider the following pseudo-metric on $\Omega$ and $\Lambda$ introduced in [10] and [7]:

$$
\begin{gathered}
\|\omega\|_{t}:=\sup _{0 \leq s \leq t}\left|\omega_{s}\right|, \\
d_{\infty}\left((t, \omega),\left(t^{\prime}, \omega^{\prime}\right)\right):=\left|t-t^{\prime}\right|^{\frac{1}{2}}+\left\|\omega_{t \wedge \cdot}-\omega_{t^{\prime} \wedge \cdot}^{\prime}\right\|_{T} .
\end{gathered}
$$


Let $C^{0}(\Lambda)$ be the set of processes $v: \Lambda \rightarrow \mathbb{R}$ that are continuous under $d_{\infty}$. We note that any $v \in C^{0}(\Lambda)$ is $\mathbb{F}$-progressively measurable. When $v$ is taking values in, say, $\mathbb{R}^{k}$, we denote it by $C^{0}\left(\Lambda ; \mathbb{R}^{k}\right)$. Let $\mathbb{S}^{d}$ denote the set of $d \times d$-symmetric matrices. We say a probability measure $\mathbb{P}$ on $\Omega$ is a semimartingale measure if $B$ is a semimartingale under $\mathbb{P}$. We now introduce the path derivatives for processes, which is due to [13] and inspired by [10].

Definition 4.3. Let $v \in C^{0}(\Lambda)$. We say $v \in C^{1,2}(\Lambda)$ if there exist $\partial_{t} v \in$ $C^{0}(\Lambda ; \mathbb{R}), \partial_{\omega} v \in C^{0}\left(\Lambda ; \mathbb{R}^{d}\right), \partial_{\omega \omega}^{2} v \in C^{0}\left(\Lambda ; \mathbb{S}^{d}\right)$ such that the following functional Itô formula holds: for any semimartingale measure $\mathbb{P}$,

$$
d v(t, \omega)=\partial_{t} v d t+\partial_{\omega} v \cdot d B_{t}+\frac{1}{2} \partial_{\omega \omega}^{2} v: d\langle B\rangle_{t}, \quad \mathbb{P} \text {-a.s. }
$$

We remark that the path derivatives $\partial_{t} v, \partial_{\omega} v, \partial_{\omega \omega}^{2} v$, if they exist, are unique.

Notice that the function $W$ in (4.12) is defined on $\Lambda \times \mathbb{R}^{d^{\prime}}$. By increasing the space dimension and viewing $y$ as the current value of the additional paths, one may easily extend all the above notions for functions on $\Lambda \times \mathbb{R}^{d^{\prime}}$ (see [13] for details).

We shall make use of the following extra assumption:

ASSUMPTION 4.4. (i) The mapping $(t, \omega) \mapsto f(t, \omega, y, z, u)$ is uniformly continuous under $d_{\infty}$, uniformly in $(y, z, u)$, and $f(t, \omega, 0,0, u)$ is bounded;

(ii) The mapping $\omega \mapsto \xi(\omega)$ is uniformly continuous under $\|\cdot\|_{T}$ and is bounded.

Under Assumption 4.4, by standard BSDE arguments one can easily show that the function $W$ defined by (4.12) is uniformly continuous and bounded. It then follows from [13] that $W$ is a viscosity solution of the following path dependent HJB equation:

$$
\left\{\begin{array}{l}
\begin{array}{l}
0=\partial_{t} W+ \\
+\inf _{(z, u)}
\end{array}\left[\frac{1}{2} \operatorname{tr}\left(\partial_{\omega \omega}^{2} W\right)\right. \\
\left.\partial_{y y}^{2} W:\left(z z^{\top}\right)+\partial_{\omega y}^{2} W \cdot z-\partial_{y} W \cdot f(t, \omega, y, z, u)\right] \\
W(T, \omega, y)=|y-\xi(\omega)|^{2}
\end{array}\right.
$$

In particular, if $W \in C^{1,2}\left(\Lambda \times \mathbb{R}^{d^{\prime}}\right)$, then $W$ is a classical solution to the above PPDE.

We shall remark though, the above PPDE is degenerate, and thus the uniqueness result of [14] does not apply here. We refer to the more recent works $[15,16]$, in which it was shown that $W$ is indeed the unique viscosity solution. We also refer to $[30,35]$ for numerical methods for PPDEs. 
4.4. Geometric DPP. We conclude this section by providing a rigorous form of the "geometric DPP" for the set valued process $\mathscr{D}(t, \omega)$ defined by $(4.9)$, that has been instrumental in the discussions of this section. Intuitively, in light of [31], we expect the following identity:

$$
\mathscr{D}\left(t_{1}, \omega\right)=\left\{y \in \mathbb{R}^{d^{\prime}}: \exists(Z, u) \in \mathbb{L}^{2}\left(\mathbb{F}^{t_{1}}, \mathbb{R}^{d^{\prime} \times d}\right) \times \mathscr{U}^{t_{1}}\right. \text { such that }
$$

$$
\left.X_{t_{2}}^{t_{1}, \omega, y, Z, u} \in \mathscr{D}\left(t_{2}, \omega \bigotimes_{t} B^{t_{1}}\right), \mathbb{P}_{0}^{t_{1}} \text {-a.s. }\right\}, \quad 0 \leq t_{1}<t_{2} \leq T .
$$

Denoting the right-hand side of $(4.20)$ by $\mathscr{D}^{\prime}\left(t_{1}, \omega\right)$, one can easily prove that $\mathscr{D}\left(t_{1}, \omega\right) \subset \mathscr{D}^{\prime}\left(t_{1}, \omega\right)$. However, the opposite inclusion is far from obvious. In what follows, we prove a weaker version of geometric DPP. We first recall (4.14) and define, for any $\varepsilon>0$,

$$
\mathscr{N}_{\varepsilon}(t, \omega):=\left\{y \in \mathbb{R}^{d^{\prime}}: W(t, \omega, y) \leq \varepsilon\right\} .
$$

It is clear that $\mathscr{N}(t, \omega)=\bigcap_{\varepsilon>0} \mathscr{N}_{\varepsilon}(t, \omega)$.

THEOREM 4.5. Under Assumptions 3.1 and 4.4, the following geometric DPP holds true:

$$
\begin{aligned}
\mathscr{N}\left(t_{1}, \omega\right)= & \bigcap_{\varepsilon>0}\left\{y \in \mathbb{R}^{d^{\prime}}: \exists\left(Z^{\varepsilon}, u^{\varepsilon}\right) \in \mathbb{L}^{2}\left(\mathbb{F}^{t_{1}}, \mathbb{R}^{d^{\prime} \times d}\right) \times \mathscr{U}^{t_{1}}\right. \text { such that } \\
& X_{t_{2}}^{t_{1}, \omega, y, Z^{\varepsilon}, u^{\varepsilon}}(\tilde{\omega}) \in \mathscr{N}_{\varepsilon}\left(t_{2}, \omega \bigotimes_{t} \tilde{\omega}\right) \\
& \left.\mathbb{P}_{0}^{t_{1}} \text {-a.e. } \tilde{\omega} \in \Omega^{t_{1}}\right\}, \quad 0 \leq t_{1}<t_{2} \leq T .
\end{aligned}
$$

ProOF. For simplicity, we assume $t_{1}=0$ and $t_{2}=t$, and let $\mathscr{N}^{\prime}\left(t_{1}, \omega\right)$ denote the right-hand side of (4.22). Noting that $\omega_{0}=0$, we shall prove that

$$
\begin{aligned}
\mathscr{N}(0,0)= & \mathscr{N}^{\prime}(0,0) \\
:= & \bigcap_{\varepsilon>0}\left\{y \in \mathbb{R}^{d^{\prime}}: \exists\left(Z^{\varepsilon}, u^{\varepsilon}\right) \in \mathbb{L}^{2}\left(\mathbb{F}, \mathbb{R}^{d^{\prime} \times d}\right) \times \mathscr{U}\right. \text { such that } \\
& \left.X_{t}^{0,0, y, Z^{\varepsilon}, u^{\varepsilon}}(\omega) \in \mathscr{N}_{\varepsilon}(t, \omega), \mathbb{P}_{0} \text {-a.e. } \omega \in \Omega\right\} .
\end{aligned}
$$

Following the arguments in [13], one shows that $W$ is uniformly continuous in $(t, \omega, y)$ with modulus of continuity function $\rho_{W}(\cdot)$, and satisfies the following DPP: recalling $\mathbb{E}:=\mathbb{E}^{\mathbb{P}_{0}}$,

$$
W(0,0, y)=\inf _{(Z, u) \in \mathbb{L}^{2}\left(\mathbb{F}, \mathbb{R}^{d^{\prime} \times d}\right) \times \mathscr{U}} \mathbb{E}\left[W\left(t, B ., X_{t}^{0,0, y, Z, u}\right)\right] .
$$


Now let $y \in \mathscr{N}^{\prime}(0,0)$. For any $\varepsilon>0$, let $\left(Z^{\varepsilon}, u^{\varepsilon}\right)$ be as in the right-hand side of (4.23). Then

$W\left(t, B, X_{t}^{0,0, y, Z^{\varepsilon}, u^{\varepsilon}}\right) \leq \varepsilon, \quad \mathbb{P}_{0}$-a.s., and thus $\quad \mathbb{E}\left[W\left(t, B, X_{t}^{0,0, y, Z^{\varepsilon}, u^{\varepsilon}}\right)\right] \leq \varepsilon$.

This, together with (4.24), implies that $W(0,0, y)=0$. Then $y \in \mathcal{N}(0,0)$, and hence $\mathscr{N}^{\prime}(0,0) \subset \mathscr{N}(0,0)$.

To see the opposite inclusion, let $y \in \mathscr{N}(0,0)$, and for any $\varepsilon>0$, choose $y_{\varepsilon} \in \mathscr{D}(0,0)$, such that $\left|y_{\varepsilon}-y\right| \leq \varepsilon$. By (4.9), let $\left(Z^{\varepsilon}, u^{\varepsilon}\right) \in \mathbb{L}^{2}\left(\mathbb{F}, \mathbb{R}^{d^{\prime} \times d}\right) \times$ $\mathscr{U}$ be such that $X_{T}^{0, \varepsilon}:=X_{T}^{0,0, y_{\varepsilon}, Z^{\varepsilon}, u^{\varepsilon}}=\xi, \mathbb{P}_{0}$-a.s. It is straightforward to see that, for $\mathbb{P}_{0}$-a.e. $\omega \in \Omega$ and $t \in[0, T],\left(Z^{\varepsilon, t, \omega}, u^{\varepsilon, t, \omega}\right) \in \mathbb{L}^{2}\left(\mathbb{F}^{t}, \mathbb{R}^{d^{\prime} \times d}\right) \times \mathscr{U}^{t}$, and $\left(X_{s}^{0, \varepsilon}\right)^{t, \omega}=X_{s}^{t, \omega, X_{t}^{0, \varepsilon}, Z^{\varepsilon, t, \omega}, u^{\varepsilon, t, \omega}}, t \leq s \leq T, \mathbb{P}_{0}^{t}$-a.s. Consequently, we have $X_{T}^{t, \omega, X_{t}^{0, \varepsilon}, Z^{\varepsilon, t, \omega}, u^{\varepsilon, t, \omega}}=\left(X_{T}^{0, \varepsilon}\right)^{t, \omega}=\xi^{t, \omega}$, and thus $X_{t}^{0, \varepsilon} \in \mathscr{D}(t, \omega)$. Now denote $X^{0, y, \varepsilon}:=X^{0,0, y, X^{\varepsilon}, u^{\varepsilon}}$, and let $\Delta X:=X^{0, \varepsilon}-X^{0, y, \varepsilon}$. Then

$$
\Delta X_{s}=y_{\varepsilon}-y+\int_{0}^{t} \alpha_{r} \Delta X_{r} d r, \quad 0 \leq s \leq t,
$$

where $\alpha$ is a bounded $\mathbb{F}$-adapted process, thanks to the Lipschitz continuity of $f$ in $y$. Then clearly $\left|\Delta X_{t}\right| \leq C\left|y_{\varepsilon}-y\right| \leq C \varepsilon$, and thus

$$
\begin{aligned}
\left|W\left(t, \omega, X_{t}^{0, y, \varepsilon}(\omega)\right)\right| & =\left|W\left(t, \omega, X_{t}^{0, y, \varepsilon}(\omega)\right)-W\left(t, \omega, X_{t}^{0, \varepsilon}(\omega)\right)\right| \\
& \leq \rho_{W}\left(\left|\Delta X_{t}(\omega)\right|\right) \leq \rho_{W}(C \varepsilon) .
\end{aligned}
$$

This implies that $X_{t}^{0,0, y, Z^{\varepsilon}, u^{\varepsilon}}(\omega) \in \mathscr{N}_{\rho(C \varepsilon)}(t, \omega)$. Since $\varepsilon>0$ is arbitrary, we obtain $y \in \mathscr{N}^{\prime}(0,0)$, and thus $\mathscr{N}(0,0) \subset \mathscr{N}^{\prime}(0,0)$.

5. The dynamic utility approach. As we have pointed out in the Introduction, as well as in Definition 3.5, one of the essential points in our scheme is to determine the "time-consistent dynamic utility" $\Phi$. We devote this section to the discussion of its existence.

5.1. The deterministic case. We begin with the case where both $f$ and $\xi$ are deterministic, and the admissible controls are also deterministic measurable functions $u \in \mathbb{L}^{0}([0, T] ; U)$. We shall still assume Assumption 3.1 holds, and try to construct $\Phi$ explicitly.

Since $\xi$ is deterministic, for $u \in \mathbb{L}^{0}([0, T] ; U)$, the solution to the $\operatorname{BSDE}(3.1)$, $\left(Y^{u}, Z^{u}\right)$, must satisfy $Z^{u} \equiv 0$. Further, if we consider the (deterministic) optimization problem:

$$
\begin{aligned}
& \Phi(t, y):=\sup _{u} \varphi\left(Y_{0}^{t, y, u}\right) \\
& \quad \text { where } Y_{s}^{t, y, u}=y+\int_{s}^{t} f\left(r, Y_{r}^{t, y, u}, 0, u_{r}\right) d r, 0 \leq s \leq t
\end{aligned}
$$


then $\Phi$ will be time consistent in the sense that it satisfies the DPP:

$$
\Phi\left(t_{2}, y\right):=\sup _{u} \Phi\left(t_{1}, Y_{t_{1}}^{t_{2}, y, u}\right), \quad \text { for } 0 \leq t_{1}<t_{2} \leq T .
$$

We shall argue that $\Phi$ is a time-consistent dynamic utility in the sense of Definition 3.5 , by identifying the required mapping $\bar{Y}$. Indeed, note that $\Phi(T, \xi)=$ $V_{0}(\xi)=\sup _{u} \varphi\left(Y_{0}^{u}\right)$, there exists $u^{\varepsilon}$ such that $\lim _{\varepsilon \rightarrow 0} \varphi\left(Y_{0}^{u^{\varepsilon}}\right)=\Phi(T, \xi)$. Denote $\bar{f}_{t}:=\sup _{u \in U}|f(t, 0,0, u)|$. By Assumption 3.1, we see that $\int_{0}^{T} \bar{f}_{t} d t<\infty$. One may easily check that

$$
\sup _{\varepsilon} \sup _{0 \leq t \leq T}\left|Y_{t}^{u^{\varepsilon}}\right| \leq C, \quad \sup _{\varepsilon}\left|Y_{t}^{u^{\varepsilon}}-Y_{s}^{u^{\varepsilon}}\right| \leq C \int_{s}^{t}\left[\bar{f}_{r}+1\right] d r, \quad 0 \leq s<t \leq T .
$$

Now, applying the Arzela-Ascoli theorem we have, possibly along a subsequence (still denoted by $u^{\varepsilon}$ ), $\lim _{\varepsilon \rightarrow 0} \sup _{0 \leq t \leq T}\left|Y_{t}^{u^{\varepsilon}}-\bar{Y}_{t}\right|=0$, and $\bar{Y}$ is an absolutely continuous function.

It is clear that $\Phi(0, y)=\varphi(y)$ and $\bar{Y}_{T}=\xi$. Further, for any two functions $u^{1}, u^{2}$, denote $u^{1} \bigotimes_{t} u^{2}:=u^{1} \mathbf{1}_{[0, t)}+u^{2} \mathbf{1}_{[t, T]}$. By stability of ODEs, one can easily check that

$$
\Phi\left(t, \bar{Y}_{t}\right)=\sup _{u} \varphi\left(Y_{0}^{t, \bar{Y}_{t}, u}\right)=\lim _{\varepsilon \rightarrow 0} \sup _{u} \varphi\left(Y_{0}^{u} \otimes_{t} u^{\varepsilon}\right) .
$$

Now on one hand, we have $\varphi\left(Y_{0}^{u \otimes_{t} u^{\varepsilon}}\right) \leq V_{0}(\xi)$ for any $u$ and $\varepsilon$. But on the other hand,

$$
\lim _{\varepsilon \rightarrow 0} \sup _{u} \varphi\left(Y_{0}^{u \otimes_{t} u^{\varepsilon}}\right) \geq \lim _{\varepsilon \rightarrow 0} \varphi\left(Y_{0}^{u^{\varepsilon} \otimes_{t} u^{\varepsilon}}\right)=\lim _{\varepsilon \rightarrow 0} \varphi\left(Y_{0}^{u^{\varepsilon}}\right)=V_{0}(\xi) .
$$

Namely, $\Phi\left(t, \bar{Y}_{t}\right)=V_{0}(\xi)$. For $0 \leq t_{1}<t_{2} \leq T$, we can follow the similar arguments to get

$$
\begin{aligned}
\sup _{u} \Phi\left(t_{1}, \mathscr{Y}_{t_{1}}^{u}\left(t_{2}, \bar{Y}_{t_{2}}\right)\right) & =\lim _{\varepsilon \rightarrow 0} \sup _{u} \Phi\left(t_{1}, \mathscr{Y}_{t_{1}}^{u}\left(t_{2}, Y_{t_{2}}^{u^{\varepsilon}}\right)\right)=\lim _{\varepsilon \rightarrow 0} \sup _{u} \Phi\left(t_{1}, Y_{t_{1}}^{u \otimes_{t_{2}} u^{\varepsilon}}\right) \\
& =\lim _{\varepsilon \rightarrow 0} \sup _{u} \sup _{u^{\prime}} \varphi\left(Y_{0}^{u^{\prime} \otimes_{t_{1}} u \bigotimes_{t_{2}} u^{\varepsilon}}\right)=V_{0}(\xi)=\Phi\left(t_{1}, \bar{Y}_{t_{1}}\right) .
\end{aligned}
$$

This verifies (3.9). To wit, $\Phi$ is indeed a time-consistent dynamic utility.

REMARK 5.1. If we denote $\tilde{\Phi}(t, y):=\Phi(T-t, y)$ and $\tilde{f}(t, y, z, u):=f(T-$ $t, y, z, u)$, then

$$
\begin{aligned}
\tilde{\Phi}(t, y)= & \sup _{u} \varphi\left(X_{T}^{t, y, u}\right), \\
& \text { where } X_{s}^{t, y, u}=y-\int_{t}^{s} \tilde{f}\left(r, X_{r}^{t, y, u}, 0, u_{r}\right) d r, t \leq s \leq T .
\end{aligned}
$$

This is a very standard (deterministic) control problem on $[0, T]$ with utility function $\varphi$. However, such a "time-change" technique would fail in the stochastic case (e.g., when $\xi$ is random), due to the adaptedness requirement. The master equation approach in Section 6 will address this issue. 
5.2. Dynamic utility via comparison principle. As we saw in Section 3, especially Examples 3.2 and 3.4, the comparison principle plays a crucial role for time consistency. In this subsection, we explore the impact of the comparison principle to the existence of the time-consistent dynamic utility $\Phi$. To this end, we propose the following stronger form of the comparison principle.

Definition 5.2. We say a mapping $\Phi: \Lambda \times \mathbb{R}^{d^{\prime}} \rightarrow \mathbb{R}$ satisfies the comparison principle if for any $t_{1}<t_{2}$ and any $\eta, \tilde{\eta} \in \mathbb{L}^{2}\left(\mathcal{F}_{t_{2}}\right), \Phi\left(t_{2}, \eta\right) \leq \Phi\left(t_{2}, \tilde{\eta}\right), \mathbb{P}_{0}$-a.s. implies that

$$
\underset{u \in \mathscr{U}}{\operatorname{esssup}} \Phi\left(t_{1}, \mathscr{Y}_{t_{1}}^{u}\left(t_{2}, \eta\right)\right) \leq \underset{u \in \mathscr{U}}{\operatorname{esssup}} \Phi\left(t_{1}, \mathscr{Y}_{t_{1}}^{u}\left(t_{2}, \tilde{\eta}\right)\right), \quad \mathbb{P}_{0} \text {-a.s. }
$$

The main result of this subsection is the following theorem.

THEOREM 5.3. Let Assumptions 3.1 and 4.4 hold and assume there exists a random field $\Phi$ satisfying the following properties:

(i) the mapping $y \mapsto \Phi(t, \omega, y)$ is continuous, for fixed $(t, \omega) \in[0, T] \times \Omega$;

(ii) $\Phi(0, \cdot, y)=\varphi(y), \mathbb{P}_{0}$-a.s.;

(iii) $\Phi$ satisfies the comparison principle in the sense of Definition 5.2.

Then $\Phi$ is a time-consistent dynamic utility in the sense of Definition 3.5.

PROOF. We shall follow the similar ideas used for the duality approach in previous sections, but here we will focus more on the measurability issue. To this end, we adjust the notation slightly. For any $t \in[0, T], \eta \in \mathbb{L}^{2}\left(\mathcal{F}_{t}, \mathbb{R}^{d^{\prime}}\right)$, $Z \in \mathbb{L}^{2}\left(\mathbb{F}, \mathbb{R}^{d^{\prime} \times d}\right)$, and $u \in \mathscr{U}$, we denote $X^{t, \eta, Z, u}$ to be the solution to the following random differential equation:

$$
\begin{aligned}
X_{s}^{t, \eta, Z, u}= & \eta-\int_{t}^{s} f\left(r, X_{r}^{t, \eta, Z, u}, Z_{r}, u_{r}\right) d r \\
& +\int_{t}^{s} Z_{r} d B_{r}, \quad t \leq s \leq T, \mathbb{P}_{0} \text {-a.s. }
\end{aligned}
$$

Clearly, (5.4) is essentially an ODE, which can be solved $\omega$-wisely. Now define

$$
\tilde{W}(t, y):=\underset{(Z, u) \in \mathbb{L}^{2}\left(\mathbb{F}, \mathbb{R}^{d^{\prime} \times d}\right) \times \mathscr{U}}{\operatorname{essinf}} \mathbb{E}_{t}\left[\left|X_{T}^{t, y, Z, u}-\xi\right|^{2}\right], \quad(t, y) \in[0, T] \times \mathbb{R}^{d^{\prime}} .
$$

Similar to (4.15) and by the uniform boundedness in Assumption 4.4, one can choose a version of $\tilde{W}$ such that

$$
\left|\tilde{W}\left(t, y_{1}\right)-\tilde{W}\left(t, y_{2}\right)\right| \leq C\left[1+\left|y_{1}\right|+\left|y_{2}\right|\right]\left|y_{1}-y_{2}\right|, \quad \mathbb{P}_{0} \text {-a.s. }
$$

Then by standard arguments one can easily show that

$$
\tilde{W}(t, \eta)=\underset{(Z, u) \in \mathbb{L}^{2}\left(\mathbb{F}, \mathbb{R}^{d^{\prime} \times d}\right) \times \mathscr{U}}{\operatorname{essinf}} \mathbb{E}_{t}\left[\left|X_{T}^{t, \eta, Z, u}-\xi\right|^{2}\right], \quad \forall \eta \in \mathbb{L}^{2}\left(\mathcal{F}_{t}, \mathbb{R}^{d^{\prime}}\right)
$$


Next, following the arguments in Theorem 4.2, one can prove the following duality results:

(D-i) If $u \in \mathscr{U}$, and $\left(Y^{u}, Z^{u}\right)$ is the solution to $\operatorname{BSDE}(3.1)$, then $\tilde{W}\left(t, Y_{t}^{u}\right)=0$, $\mathbb{P}_{0}$-a.s.;

(D-ii) If $\eta \in \mathbb{L}^{0}\left(\mathcal{F}_{t}, \mathbb{R}^{d^{\prime}}\right)$ is such that $\tilde{W}(t, \eta)=0, \mathbb{P}_{0}$-a.s., then $\eta \in \mathbb{L}^{2}\left(\mathcal{F}_{t}, \mathbb{R}^{d^{\prime}}\right)$. Furthermore, for any $\varepsilon>0$, there exists $u^{\varepsilon} \in \mathscr{U}$, such that

$$
\left|Y_{t}^{u^{\varepsilon}}-\eta\right| \leq C \varepsilon, \quad \text { and } \quad \lim _{\varepsilon \rightarrow 0} \Phi\left(t, Y_{t}^{u^{\varepsilon}}\right)=\Phi(t, \eta), \quad \mathbb{P}_{0} \text {-a.s. }
$$

We now construct the family of maximizers $\left\{\bar{Y}_{t}\right\}$. For each fixed $t \in[0, T]$, denote $\tilde{\mathscr{N}}_{t}:=\left\{(\omega, y) \in \Omega \times \mathbb{R}^{d^{\prime}}: \tilde{W}(t, \omega, y)=0\right\}$ and $\tilde{\mathscr{N}}_{t}(\omega):=\left\{y \in \mathbb{R}^{d^{\prime}}:(\omega, y) \in\right.$ $\left.\tilde{\mathscr{N}}_{t}\right\}$. Then $\tilde{\mathscr{N}}_{t}$ is $\mathcal{F}_{t} \times \mathcal{B}\left(\mathbb{R}^{d^{\prime}}\right)$-measurable, and for $\mathbb{P}_{0}$-a.e. $\omega \in \Omega, \tilde{\mathscr{N}}_{t}(\omega)$ is closed and bounded, whence compact. Define $\bar{\Phi}_{t}(\omega):=\sup _{y \in \tilde{\mathscr{N}}(\omega)} \Phi(t, \omega, y)$, and denote

$$
\mathscr{M}_{t}:=\left\{(\omega, y) \in \tilde{\mathscr{N}}_{t}: \bar{\Phi}_{t}(\omega)=\Phi(t, \omega, y)\right\}, \quad \mathscr{M}_{t}(\omega):=\mathscr{M}_{t} \cap \tilde{\mathscr{N}}_{t}(\omega) .
$$

Then it is easy to see that $\bar{\Phi}_{t}$ is $\mathcal{F}_{t}$-measurable and $\mathscr{M}_{t}$ is $\mathcal{F}_{t} \times \mathcal{B}\left(\mathbb{R}^{d^{\prime}}\right)$-measurable. Moreover, the continuity of $\Phi$ in $y$ implies that $\mathscr{M}_{t}(\omega)$ is nonempty and compact, for $\mathbb{P}_{0}$-a.e. $\omega \in \Omega$. Now let $\bar{Y}_{t}(\omega)$ be the (unique) maximum point of $\mathscr{M}_{t}(\omega)$ under the following order on $\mathbb{R}^{d^{\prime}}$ :

$$
y<y^{\prime} \Longleftrightarrow \text { for some } i=1, \ldots, d^{\prime}, y_{j}=y_{j}^{\prime}, j=1, \ldots, i-1 \text {, and } y_{i}<y_{i}^{\prime} \text {. }
$$

Then clearly $\bar{Y}_{t}$ is $\mathcal{F}_{t}$-measurable, and $\bar{Y}_{t}(\omega) \in \mathcal{M}_{t}(\omega)$.

We now verify that $\bar{Y}$ satisfies all the requirements in Definition 3.5. First, it is clear that $\tilde{\mathscr{N}}_{T}(\omega)=\{\xi(\omega)\}$, and thus $\bar{Y}_{T}(\omega)=\xi(\omega)$. We next show that

$$
\bar{Y}_{t} \in \mathbb{L}^{2}\left(\mathcal{F}_{t}, \mathbb{R}^{d^{\prime}}\right) \quad \text { and } \Phi\left(t, \bar{Y}_{t}\right)=\underset{u \in \mathscr{U}}{\operatorname{esssup}} \Phi\left(t, Y_{t}^{u}\right) .
$$

Indeed, for any $u \in \mathscr{U}$, by the duality result (D-i) above we have $\tilde{W}\left(t, Y_{t}^{u}\right)=0$. That is, $Y_{t}^{u}(\omega) \in \tilde{\mathscr{N}}_{t}(\omega)$, and thus $\Phi\left(t, \omega, Y_{t}^{u}(\omega)\right) \leq \bar{\Phi}_{t}(\omega)=\Phi\left(t, \omega, \bar{Y}_{t}(\omega)\right.$, for $\mathbb{P}_{0}$-a.e. $\omega \in \Omega$. Conversely, since $\bar{Y}_{t}(\omega) \in \mathscr{M}_{t}(\omega) \subset \tilde{\mathscr{N}}_{t}(\omega)$, we see that $\tilde{W}\left(t, \omega, \bar{Y}_{t}(\omega)\right)=0$ for $\mathbb{P}_{0}$-a.e. $\omega$. Then by the duality result (D-ii) and (5.7) we prove (5.8) immediately.

It remains to verify the DPP (3.9). Note that for any $u^{0} \in \mathscr{U},(5.8)$ implies that $\Phi\left(t_{2}, Y_{t_{2}}^{u^{0}}\right) \leq \Phi\left(t_{2}, \bar{Y}_{t_{2}}\right), \mathbb{P}_{0}$-a.s. Then it follows from the comparison principle (5.3) that

$$
\underset{u \in \mathscr{U}}{\operatorname{esssup}} \Phi\left(t_{1}, \mathscr{Y}_{t_{1}}^{u}\left(t_{2}, Y_{t_{2}}^{u^{0}}\right)\right) \leq \underset{u \in \mathscr{U}}{\operatorname{esssup}} \Phi\left(t_{1}, \mathscr{Y}_{t_{1}}^{u}\left(t_{2}, \bar{Y}_{t_{2}}\right)\right), \quad \mathbb{P}_{0} \text {-a.s. }
$$

Note that by definition $\mathscr{Y}_{t_{1}}^{u}\left(t_{2}, Y_{t_{2}}^{u^{0}}\right)=Y_{t_{1}}^{u \otimes_{t_{2}} u^{0}}$, then clearly

$$
\Phi\left(t_{1}, \bar{Y}_{t_{1}}\right)=\underset{u \in \mathscr{U}}{\operatorname{esssup}} \Phi\left(t_{1}, Y_{t_{1}}^{u}\right) \leq \underset{u \in \mathscr{U}}{\operatorname{esssup}} \Phi\left(t_{1}, \mathscr{Y}_{t_{1}}^{u}\left(t_{2}, \bar{Y}_{t_{2}}\right)\right), \quad \mathbb{P}_{0} \text {-a.s. }
$$


On the other hand, again by (5.8), there exist $\left\{u^{\varepsilon}\right\}_{\varepsilon>0} \subseteq \mathscr{U}$ such that $\left|Y_{t_{2}}^{u^{\varepsilon}}-\bar{Y}_{t_{2}}\right| \leq$ $C \varepsilon, \mathbb{P}_{0}$-a.s. Then for any $u \in \mathscr{U}$, by the stability of BSDE and the continuity of $\Phi$ in $y$,

$\Phi\left(t_{1}, \mathscr{Y}_{t_{1}}^{u}\left(t_{2}, \bar{Y}_{t_{2}}\right)\right)=\lim _{\varepsilon \rightarrow 0} \Phi\left(t_{1}, \mathscr{Y}_{t_{1}}^{u}\left(t_{2}, Y_{t_{2}}^{u^{\varepsilon}}\right)\right)=\lim _{\varepsilon \rightarrow 0} \Phi\left(t_{1}, Y_{t_{1}}^{u \bigotimes_{t_{2}} u^{\varepsilon}}\right) \leq \Phi\left(t_{1}, \bar{Y}_{t_{1}}\right)$.

Since $u \in \mathscr{U}$ is arbitrary, we obtain (3.9), completing the proof.

5.3. The linear case. While Theorem 5.3 gives a guiding principle for finding the time-consistent dynamic utility function, it would be extremely desirable to see if a function satisfying the comparison principle (5.3) does exist. In this subsection, we shall construct an explicit example, in the case when both the BSDE (3.1) and function $\varphi$ are linear. Our construction follows the dimension reduction technique in [24].

THEOREM 5.4. Let Assumption 3.1 hold and assume that the coefficients $f$ and $\varphi$ are of the following linear form:

$$
\left\{\begin{array}{l}
f_{i}(t, \omega, y, z, u)=\sum_{j=1}^{d^{\prime}}\left[\alpha_{t}^{i, j}(\omega) y_{j}+\beta_{t}^{i, j}(\omega) \cdot z_{j}\right]+c_{i}(t, \omega, u), \\
i=1, \ldots, d^{\prime} \\
\varphi(y)=\sum_{i=1}^{d^{\prime}} a_{i} y_{i}
\end{array}\right.
$$

Then there exists a random field $\Phi$ satisfying the comparison principle (5.3), which takes the following linear form:

$$
\Phi(t, \omega, y):=\sum_{i=1}^{d^{\prime}} A_{t}^{i}(\omega) y_{i} \quad \text { with } A_{0}^{i}=a_{i}
$$

Proof. We first note that if $d^{\prime}=1$, then the BSDE (3.1) is 1-dimensional, thus the comparison principle holds. Further since $\varphi$ is linear, whence monotone, thus the problem is time consistent and the theorem becomes trivial. We shall thus concentrate on multidimensional cases. Note also that for $d^{\prime} \geq 2$, following an inductional arguments as illustrated in [24], Section 4.1, we need only prove the case $d^{\prime}=2$. We shall split the proof (assuming $d^{\prime}=2$ ) in three steps.

Step 1 . We begin by a heuristic argument which will lead us to the desired properties of the processes $A^{1}$ and $A^{2}$. For convenience, we shall assume that $A^{1}$ and $A^{2}$ take the form of the Itô process:

$$
A_{t}^{i}=a_{i}+\int_{0}^{t} b_{s}^{i} d s+\int_{0}^{t} \sigma_{s}^{i} d B_{s}, \quad i=1,2 .
$$


For any $u \in \mathscr{U}$ and the corresponding solution $\left(Y^{u}, Z^{u}\right)$, we define

$$
\begin{aligned}
& \hat{Y}_{t}^{u}:=\Phi\left(t, \cdot, Y_{t}^{u}\right):=\sum_{i=1}^{2} A_{t}^{i} Y_{t}^{i, u}, \\
& \hat{Z}_{t}^{u}:=\sum_{i=1}^{2}\left[A_{t}^{i} Z_{t}^{i, u}+\sigma_{t}^{i} Y_{t}^{i, u}\right], \quad t \in[0, T] .
\end{aligned}
$$

We hope to find a pair of processes $\left(A^{1}, A^{2}\right)$ so that $\left(\hat{Y}^{u}, \hat{Z}^{u}\right)$ satisfy a onedimensional BSDE, so as to reduce the problem to the case $d^{\prime}=1$.

To this end, we first assume $A_{t}^{2} \equiv a_{2} \neq 0,0 \leq t \leq T$, Then an easy application of Itô's formula and some direct computations lead us to

$$
\begin{aligned}
d \hat{Y}_{t}^{u}= & {\left[A_{t}^{1} d Y_{t}^{1, u}+Y_{t}^{1, u} d A_{t}^{1}+\sigma_{t}^{1} Z_{t}^{1, u} d t+a_{2} d Y_{t}^{2, u}\right] } \\
= & -\left[A_{t}^{1} \sum_{j=1}^{2}\left[\alpha_{t}^{1, j} Y_{t}^{j, u}+\beta_{t}^{1, j} Z_{t}^{j, u}\right]+A_{t}^{1} c_{1}\left(t, u_{t}\right)\right. \\
& \left.+a_{2} \sum_{j=1}^{2}\left[\alpha_{t}^{2, j} Y_{t}^{j, u}+\beta_{t}^{2, j} Z_{t}^{j, u}\right]+a_{2} c_{2}\left(t, u_{t}\right)-\left[b_{t}^{1} Y_{t}^{1, u}+\sigma_{t}^{1} Z_{t}^{1, u}\right]\right] d t \\
& +\left[A_{t}^{1} Z_{t}^{1, u}+\sigma_{t}^{1} Y_{t}^{1, u}+a_{2} Z_{t}^{2, u}\right] d B_{t} .
\end{aligned}
$$

Note that in this case $b^{2}=\sigma^{2}=0$, we see from (5.12) that $A_{t}^{1} Z_{t}^{1, u}+\sigma_{t}^{1} Y_{t}^{1, u}+$ $a_{2} Z_{t}^{2, u}=\hat{Z}_{t}^{u}$, and thus

$$
Y_{t}^{2, u}=a_{2}^{-1}\left[\hat{Y}_{t}^{u}-A_{t}^{1} Y_{t}^{1, u}\right], \quad Z_{t}^{2, u}=a_{2}^{-1}\left[\hat{Z}_{t}^{u}-\sigma_{t}^{1} Y_{t}^{1, u}-A_{t}^{1} Z_{t}^{1, u}\right] .
$$

Plugging these into (5.13) and reorganizing terms yieldsa

$$
\begin{aligned}
-d \hat{Y}_{t}^{u}+ & \hat{Z}_{t}^{u} d B_{t} \\
= & {\left[\left[A_{t}^{1} \alpha_{t}^{1,1}+a_{2} \alpha_{t}^{2,1}-b_{t}^{1}\right] Y_{t}^{1, u}+\left[A_{t}^{1} \beta_{t}^{1,1}+a_{2} \beta_{t}^{2,1}-\sigma_{t}^{1}\right] Z_{t}^{1, u}\right.} \\
& +A_{t}^{1} c_{1}\left(t, u_{t}\right)+a_{2} c_{2}\left(t, u_{t}\right)+\left[A_{t}^{1} \alpha_{t}^{1,2}+a_{2} \alpha_{t}^{2,2}\right] a_{2}^{-1}\left[\hat{Y}_{t}^{u}-A_{t}^{1} Y_{t}^{1, u}\right] \\
& \left.+\left[A_{t}^{1} \beta_{t}^{1,2}+a_{2} \beta_{t}^{2,2}\right] a_{2}^{-1}\left[\hat{Z}_{t}^{u}-\sigma_{t}^{1} Y_{t}^{1, u}-A_{t}^{1} Z_{t}^{1, u}\right]\right] d t \\
= & {\left[a_{2}^{-1}\left[A_{t}^{1} \alpha_{t}^{1,2}+a_{2} \alpha_{t}^{2,2}\right] \hat{Y}_{t}^{u}+a_{2}^{-1}\left[A_{t}^{1} \beta_{t}^{1,2}+a_{2} \beta_{t}^{2,2}\right] \hat{Z}_{t}^{u}\right.} \\
& \left.+A_{t}^{1} c_{1}\left(t, u_{t}\right)+a_{2} c_{2}\left(t, u_{t}\right)+\Theta_{t} Y_{t}^{1, u}+\Gamma_{t} Z_{t}^{1, u}\right] d t,
\end{aligned}
$$

where

$$
\begin{aligned}
\Theta_{t}:= & {\left[A_{t}^{1} \alpha_{t}^{1,1}+a_{2} \alpha_{t}^{2,1}-b_{t}^{1}\right]-a_{2}^{-1} A_{t}^{1}\left[A_{t}^{1} \alpha_{t}^{1,2}+a_{2} \alpha_{t}^{2,2}\right] } \\
& -a_{2}^{-1} \sigma_{t}^{1}\left[A_{t}^{1} \beta_{t}^{1,2}+a_{2} \beta_{t}^{2,2}\right] ; \\
\Gamma_{t}:= & {\left[A_{t}^{1} \beta_{t}^{1,1}+a_{2} \beta_{t}^{2,1}-\sigma_{t}^{1}\right]-a_{2}^{-1} A_{t}^{1}\left[A_{t}^{1} \beta_{t}^{1,2}+a_{2} \beta_{t}^{2,2}\right] . }
\end{aligned}
$$


Now setting $\Theta_{t} \equiv \Gamma_{t} \equiv 0$, we see that (5.14) becomes a linear BSDE for $\left(\hat{Y}^{u}, \hat{Z}^{u}\right)$. But this can be done by simply solving

$$
\begin{aligned}
\sigma_{t}^{1} & :=\left[A_{t}^{1} \beta_{t}^{1,1}+a_{2} \beta_{t}^{2,1}\right]-a_{2}^{-1} A_{t}^{1}\left[A_{t}^{1} \beta_{t}^{1,2}+a_{2} \beta_{t}^{2,2}\right] \\
b_{t}^{1} & :=\left[A_{t}^{1} \alpha_{t}^{1,1}+a_{2} \alpha_{t}^{2,1}\right]-a_{2}^{-1} A_{t}^{1}\left[A_{t}^{1} \alpha_{t}^{1,2}+a_{2} \alpha_{t}^{2,2}\right]-a_{2}^{-1} \sigma_{t}^{1}\left[A_{t}^{1} \beta_{t}^{1,2}+a_{2} \beta_{t}^{2,2}\right] .
\end{aligned}
$$

Note that the processes $b^{1}$ and $\sigma^{1}$ can be easily written as functions of the process $a_{2}^{-1} A^{1}$ by setting $b_{t}^{1}=a_{2} \hat{b}_{1}\left(t, \omega, a_{2}^{-1} A_{t}^{1}\right)$ and $\sigma_{t}^{1}=a_{2} \hat{\sigma}_{1}\left(t, \omega, a_{2}^{-1} A_{t}^{1}\right)$, where

$$
\begin{aligned}
\hat{\sigma}_{1}(t, x):= & -\beta_{t}^{1,2}|x|^{2}+\left[\beta_{t}^{1,1}-\beta_{t}^{2,2}\right] x+\beta_{t}^{2,1} ; \\
\hat{b}_{1}(t, x):= & \left|\beta_{t}^{1,2}\right|^{2} x^{3}-\left[\alpha^{1,2}+\beta^{1,2}\left[\beta^{1,1}-\beta^{2,2}\right]-\beta^{1,2} \beta^{22}\right] x^{2} \\
& +\left[\alpha_{t}^{1,1}-\alpha_{t}^{2,2}-\beta^{2,2}\left[\beta_{t}^{1,1}-\beta_{t}^{2,2}\right]-\beta_{t}^{1,2} \beta_{t}^{2,1}\right] x \\
& +\left[\alpha^{2,1}-\beta_{t}^{2,1} \beta_{t}^{2,2}\right] .
\end{aligned}
$$

Plugging this into (5.11), we obtain an SDE for $A_{t}^{1}$ :

$$
A_{t}^{1} / a_{2}=a_{1} / a_{2}+\int_{0}^{t} \hat{b}_{1}\left(s, a_{2}^{-1} A_{s}^{1}\right) d s+\int_{0}^{t} \hat{\sigma}_{1}\left(s, a_{2}^{-1} A_{s}^{1}\right) d B_{s}, \quad t \geq 0
$$

We should note that since the coefficients $\hat{\sigma}$ has quadratic growth in $A_{t}^{1}$ and $\hat{b}$ has triple growth in $A_{t}^{1}$, the $\operatorname{SDE}(5.16)$ is a Ricatti equation in general sense and has only local solutions. However, if (5.16) is solvable, which we shall argue rigorously in the next step, then we will see that the $\Phi(t, \cdot)$ defined by $(5.10)$ satisfies the comparison principle (5.3).

Step 2. We now substantiate the idea in Step 1 rigorously. If $a_{1}=a_{2}=0$, then clearly $V_{0}(\xi)=0$ and there is nothing to prove. From now on, we assume without loss of generality that $\left|a_{1}\right| \leq\left|a_{2}\right|$ and $a_{2} \neq 0$. Denote $\tau_{0}:=0$. Recall (5.16) and consider the following SDE:

$$
\begin{aligned}
\hat{A}_{t}^{1}= & a_{1} / a_{2}+\int_{0}^{t} \hat{b}_{1}\left(s,[-2] \vee \hat{A}_{s}^{1} \wedge 2\right) d s \\
& +\int_{0}^{t} \hat{\sigma}_{1}\left(s,[-2] \vee \hat{A}_{s}^{1} \wedge 2\right) d B_{s}, \quad t \in[0, T] .
\end{aligned}
$$

Clearly, $\hat{A}^{1}$ has global solution. Define $\tau_{1}:=\inf \left\{t \geq 0:\left|\hat{A}_{t}^{1}\right| \geq 2\right\} \wedge T$. Then

$$
\hat{A}_{t}^{1}=a_{1} / a_{2}+\int_{0}^{t} \hat{b}_{1}\left(s, \hat{A}_{s}^{1}\right) d s+\int_{0}^{t} \hat{\sigma}_{1}\left(s, \hat{A}_{s}^{1}\right) d B_{s}, \quad \tau_{0} \leq t \leq \tau_{1} .
$$

We now set $A_{t}^{1}:=a_{2} \hat{A}_{t}^{1}$ and $A_{t}^{2}:=a_{2}$, for $\tau_{0} \leq t \leq \tau_{1}$. Then, noting that $\left|\hat{A}_{\tau_{1}}^{1}\right|=2$ [or $\left|\left(\hat{A}_{\tau_{1}}^{1}\right)^{-1}\right|=\frac{1}{2}$ ] when $\tau_{1}<T$ and reversing the roles of $A^{1}$ and $A^{2}$ as in Step 1 we can then obtain coefficients $\hat{b}_{2}, \hat{\sigma}_{2}$ completely symmetric as those in (5.15), and an SDE on $\left[\tau_{1}, T\right]$ :

$$
\hat{A}_{t}^{2}=\left(\hat{A}_{\tau_{1}}^{1}\right)^{-1}+\int_{\tau_{1}}^{t} \hat{b}_{2}\left(s,[-2] \vee \hat{A}_{s}^{2} \wedge 2\right) d s+\int_{0}^{t} \hat{\sigma}_{2}\left(s,[-2] \vee \hat{A}_{s}^{2} \wedge 2\right) d B_{s} .
$$


Similarly, $\hat{A}^{2}$ has global solution, and that

$$
\hat{A}_{t}^{2}=\left(\hat{A}_{\tau_{1}}^{1}\right)^{-1}+\int_{\tau_{1}}^{t} \hat{b}_{2}\left(s, \hat{A}_{s}^{2}\right) d s+\int_{0}^{t} \hat{\sigma}_{2}\left(s, \hat{A}_{s}^{2}\right) d B_{s}, \quad \tau_{1} \leq t \leq \tau_{2}
$$

where $\tau_{2}:=\inf \left\{t \geq \tau_{1}:\left|\hat{A}_{t}^{2} / A_{\tau_{1}}^{1}\right| \geq 2\right\} \wedge T$. We then define $A_{t}^{1}:=A_{\tau_{1}}^{1}$, and $A_{t}^{2}:=$ $A_{\tau_{1}}^{1} \hat{A}_{t}^{2}$, for $\tau_{1} \leq t \leq \tau_{2}$. Note that since $A_{\tau_{1}}^{1} \hat{A}_{\tau_{1}}^{2}=A_{\tau_{1}}^{1}\left(\hat{A}_{\tau_{1}}^{1}\right)^{-1}=a_{2}=A_{\tau_{1}}^{2}$, both $A^{1}$ and $A^{2}$ are continuous at $\tau_{1}$.

Now repeating the arguments, we may define, for $n \geq 1$, processes $\left\{\hat{A}^{n}\right\}$ and stopping times $0=\tau_{0} \leq \tau_{1} \leq \tau_{n} \cdots$, such that

$$
\begin{aligned}
& \hat{A}_{t}^{2 n}=\left(\hat{A}_{\tau_{2 n-1}}^{2 n-1}\right)^{-1}+\int_{\tau_{2 n-1}}^{t} \hat{b}_{2}\left(s, \hat{A}_{s}^{2 n}\right) d s+\int_{\tau_{2 n-1}}^{t} \hat{\sigma}_{2}\left(s, \hat{A}_{s}^{2 n}\right) d B_{s}, \\
& \tau_{2 n-1} \leq t \leq \tau_{2 n} ; \\
& \hat{A}_{t}^{2 n+1}=\left(\hat{A}_{\tau_{2 n}}^{2 n}\right)^{-1}+\int_{\tau_{2 n}}^{t} \hat{b}_{1}\left(s, \hat{A}_{s}^{2 n+1}\right) d s+\int_{\tau_{2 n}}^{t} \hat{\sigma}_{1}\left(s, \hat{A}_{s}^{2 n+1}\right) d B_{s}, \\
& \tau_{2 n} \leq t \leq \tau_{2 n+1} .
\end{aligned}
$$

Furthermore, for all $n \geq 1$, it holds that $\left|\hat{A}_{t}^{n}\right|<2, \tau_{n-1} \leq t<\tau_{n}$, and $\left|\hat{A}_{\tau_{n}}^{n}\right|=2$ on $\left\{\tau_{n}<T\right\}$. The rest of the argument will be based on the following fact, which will be validated in the next step:

$$
\mathbb{P}_{0}\left(\bigcup_{n \geq 1}\left\{\tau_{n}=T\right\}\right)=1
$$

Assuming (5.20), we can now define continuous processes $A^{1}, A^{2}$ on $[0, T]$ :

$$
\begin{aligned}
& A_{t}^{1}:=A_{\tau_{2 n-1}}^{1}, \quad A_{t}^{2}:=A_{\tau_{2 n-1}}^{1} \hat{A}_{t}^{2 n}, \quad \tau_{2 n-1}<t \leq \tau_{2 n} ; \\
& A_{t}^{1}:=A_{\tau_{2 n}}^{2} \hat{A}_{t}^{2 n+1}, \quad A_{t}^{2}:=A_{\tau_{2 n}}^{2}, \quad \tau_{2 n}<t \leq \tau_{2 n+1} .
\end{aligned}
$$

Now define $\Phi$ by (5.10) and $\left(\hat{Y}^{u}, \hat{Z}^{u}\right)$ by (5.12). We can rewrite (5.13) as

$$
d \hat{Y}_{t}^{u}=-\left[\hat{\alpha}_{t} \hat{Y}_{t}^{u}+\hat{\beta}_{t} \hat{Z}_{t}^{u}+\sum_{i=1}^{2} A_{t}^{i} c_{i}\left(t, u_{t}\right)\right] d t+\hat{Z}_{t}^{u} d B_{t}, \quad 0 \leq t \leq T,
$$

where

$$
\begin{aligned}
& \hat{\alpha}_{t}= \begin{cases}\alpha_{t}^{1,2} \hat{A}_{t}^{2 n+1}+\alpha_{t}^{2,2} & \text { on }\left[\tau_{2 n}, \tau_{2 n+1}\right], \\
\alpha_{t}^{2,1} \hat{A}_{t}^{2 n}+\alpha_{t}^{1,1} & \text { on }\left[\tau_{2 n-1}, \tau_{2 n}\right] ;\end{cases} \\
& \hat{\beta}_{t}= \begin{cases}\beta_{t}^{1,2} \hat{A}_{t}^{2 n+1}+\beta_{t}^{2,2} & \text { on }\left[\tau_{2 n}, \tau_{2 n+1}\right], \\
\beta_{t}^{2,1} \hat{A}_{t}^{2 n}+\beta_{t}^{1,1} & \text { on }\left[\tau_{2 n-1}, \tau_{2 n}\right] .\end{cases}
\end{aligned}
$$


Note that $\left|\hat{A}_{t}^{2 n+1}\right| \leq 2$ on $\tau_{2 n} \leq t \leq \tau_{2 n+1}$ and $\left|\hat{A}_{t}^{2 n}\right| \leq 2$ on $\tau_{2 n-1} \leq t \leq \tau_{2 n}$; both $\hat{\alpha}, \hat{\beta}$ are bounded. Now denoting $\hat{Y}_{t}^{u}(\xi)$ to emphasize the dependence on the terminal condition $\xi$, it follows from the definition (5.12) and the comparison of BSDEs that

$$
\begin{aligned}
\Phi(T, \xi) \leq & \Phi(T, \tilde{\xi}) \\
& \Longrightarrow \quad \hat{Y}_{t}^{u}(\xi) \leq \hat{Y}_{t}^{u}(\tilde{\xi}), \quad \forall u \in \mathscr{U} \\
& \Longrightarrow \quad \operatorname{esssup}_{u \in \mathscr{U}} \Phi\left(t, \mathscr{Y}_{t}^{u}(T, \xi)\right) \leq \underset{u \in \mathscr{U}}{\operatorname{esssup}} \Phi\left(t, \mathscr{Y}_{t}^{u}(T, \tilde{\xi})\right), \quad \mathbb{P}_{0} \text {-a.s. }
\end{aligned}
$$

The same argument can be used to treat any subinterval $\left[t_{1}, t_{2}\right]$, proving (5.3).

Step 3. It remains to prove (5.20). Fix some $\delta>0$. Note that $\left|a_{1} / a_{2}\right| \leq 1$. By (5.17) and standard estimates for SDEs, we can easily check that $\mathbb{E}\left[\sup _{0 \leq t \leq T}\left|\hat{A}_{t}^{1}\right|^{2}\right] \leq C$. Thus,

$$
\begin{aligned}
\mathbb{P}_{0}\left(\tau_{1}\right. & <T \wedge \delta) \\
& \leq \mathbb{P}_{0}\left(\sup _{0 \leq t \leq \delta}\left|\hat{A}_{t}^{1}\right| \geq 2\right) \\
& \leq \mathbb{P}_{0}\left(\sup _{0 \leq t \leq \delta}\left|\hat{A}_{t}^{1}-\hat{A}_{0}^{1}\right| \geq 1\right) \leq \mathbb{E}\left[\sup _{0 \leq t \leq \delta}\left|\hat{A}_{t}^{1}-\hat{A}_{0}^{1}\right|^{2}\right] \\
& \leq C \mathbb{E}\left[\int_{0}^{\delta}\left|\hat{b}_{1}\left(s,[-2] \vee \hat{A}_{s}^{1} \wedge 2\right)\right|^{2} d s+\int_{0}^{\delta}\left|\hat{\sigma}_{1}\left(s,[-2] \vee \hat{A}_{s}^{1} \wedge 2\right)\right|^{2} d s\right] \\
& \leq C \delta .
\end{aligned}
$$

Now setting $\delta:=\frac{1}{2 C}$, so that

$$
\mathbb{P}_{0}\left(\tau_{1}<T, \tau_{1} \leq \delta\right) \leq \frac{1}{2} .
$$

Similarly, noting that $\left|\hat{A}_{\tau_{1}}^{2}\right|=\frac{1}{2}$ and $\left|\hat{A}_{\tau_{2}}^{2}\right|=2$ on $\left\{\tau_{2}<T\right\}$, we have

$$
\mathbb{P}_{0}\left(\tau_{2}<T \wedge\left(\tau_{1}+\delta\right) \mid \mathcal{F}_{\tau_{1}}\right) \leq \frac{1}{2}
$$

Repeating the arguments, for any $n$ one shows that

$$
\mathbb{P}_{0}\left(\tau_{n+1}<T \wedge\left(\tau_{n}+\delta\right) \mid \mathcal{F}_{\tau_{n}}\right) \leq \frac{1}{2} .
$$

We shall prove (5.20) by arguing that $\mathbb{P}_{0}\left\{\left(\bigcup_{n \geq 1}\left\{\tau_{n}=T\right\}\right)^{c}\right\}=\mathbb{P}_{0}\left\{\bigcap_{n \geq 1}\left\{\tau_{n}<\right.\right.$ $T\}\}=0$. But since $\tau_{n}$ 's are increasing, this amounts to saying that $\lim _{n \rightarrow \infty} \overline{\mathbb{P}}_{0}\left\{\tau_{n}<\right.$ $T\}=0$. Now for the given $\delta$, we can assume that $m \delta<T \leq(m+1) \delta$, for some $m \in \mathbb{N}$. We claim the following much stronger result, which obviously implies (5.20): for any $n \geq 1$,

$$
\mathbb{P}_{0}\left(\tau_{n}<T\right) \leq \frac{(2 n)^{m}}{2^{n}}, \quad \text { whenever } m \delta<T \leq(m+1) \delta .
$$


We shall prove (5.26) by induction on $m$. First, if $m=0$, namely $0<T \leq \delta$, then

$$
\begin{aligned}
\mathbb{P}_{0}\left(\tau_{n}<T\right) & =\mathbb{P}_{0}\left(\tau_{n}<T, \tau_{1} \leq \delta\right)=\mathbb{P}_{0}\left(\tau_{1}<T, \tau_{1} \leq \delta\right) \mathbb{P}_{0}\left(\tau_{n}<T \mid \mathcal{F}_{\tau_{1}}, \tau_{1}<T\right) \\
& \leq \frac{1}{2} \mathbb{P}_{0}\left(\tau_{n}<T \mid \mathcal{F}_{\tau_{1}}, \tau_{1}<T\right)
\end{aligned}
$$

thanks to (5.23). By (5.25), for $k<n$ we have

$$
\mathbb{P}_{0}\left(\tau_{n}<T \mid \mathcal{F}_{\tau_{k-1}}, \tau_{k-1}<T\right) \leq \frac{1}{2} \mathbb{P}_{0}\left(\tau_{n}<T \mid \mathcal{F}_{\tau_{k}}, \tau_{k}<T\right) .
$$

Then by induction we see that

$$
\mathbb{P}_{0}\left(\tau_{n}<T\right) \leq \frac{1}{2^{n-1}} \mathbb{P}_{0}\left(\tau_{n}<T \mid \mathcal{F}_{\tau_{n-1}}, \tau_{n-1}<T\right) \leq \frac{1}{2^{n}}
$$

proving (5.26) for $m=0$.

Assume (5.26) holds for $m-1$ and we shall prove it for $m$. By (5.23), we have

$$
\begin{aligned}
\mathbb{P}_{0}\left(\tau_{n}<T\right)= & \mathbb{P}_{0}\left(\tau_{n}<T, \tau_{1} \leq \delta\right)+\mathbb{P}_{0}\left(\tau_{n}<T, \tau_{1}>\delta\right) \\
\leq & \mathbb{P}_{0}\left(\tau_{1}<T, \tau_{1} \leq \delta\right) \mathbb{P}_{0}\left(\tau_{n}<T \mid \mathcal{F}_{\tau_{1}}, \tau_{1}<T\right) \\
& +\mathbb{P}_{0}\left(\tau_{n}<T, \tau_{n}-\tau_{1}<T-\delta\right) \\
\leq & \frac{1}{2} \mathbb{P}_{0}\left(\tau_{n}<T \mid \mathcal{F}_{\tau_{1}}, \tau_{1}<T\right)+\mathbb{P}_{0}\left(\tau_{n}<T, \tau_{n}-\tau_{1}<T-\delta\right) .
\end{aligned}
$$

Note that $(m-1) \delta<T-\delta \leq m \delta$, then the inductional hypothesis implies that

$$
\mathbb{P}_{0}\left(\tau_{n}<T, \tau_{n}-\tau_{1}<T-\delta\right) \leq \frac{(2 n-2)^{m-1}}{2^{n-1}},
$$

and thus

$$
\mathbb{P}_{0}\left(\tau_{n}<T\right) \leq \frac{1}{2} \mathbb{P}_{0}\left(\tau_{n}<T \mid \mathcal{F}_{\tau_{1}}, \tau_{1}<T\right)+\frac{(2 n-2)^{m-1}}{2^{n-1}} .
$$

By (5.25), for $k<n$ we have

$$
\mathbb{P}_{0}\left(\tau_{n}<T \mid \mathcal{F}_{\tau_{k-1}}, \tau_{k-1}<T\right) \leq \frac{1}{2} \mathbb{P}_{0}\left(\tau_{n}<T \mid \mathcal{F}_{\tau_{k}}, \tau_{k}<T\right)+\frac{(2 n-2 k)^{m-1}}{2^{n-k}}
$$

Then by induction we have

$$
\mathbb{P}_{0}\left(\tau_{n}<T\right) \leq \frac{1}{2^{n}}+\sum_{k=1}^{n-1} \frac{(2 k)^{m-1}}{2^{n-1}}=\frac{1+2 \sum_{k=1}^{n-1}(2 k)^{m-1}}{2^{n}} .
$$

It is straightforward to check that $1+2 \sum_{k=1}^{n-1}(2 k)^{m-1} \leq(2 n)^{m}$, proving (5.20), whence the theorem. 
6. The master equation approach. In this section, we deviate from the dynamic utility $\Phi$ and attack the value function $V_{0}(\xi)$ from a different direction. We begin by noticing that, unlike the forward stochastic control problem where the value function depends on the "initial data," in our problem the value $V_{0}(\xi)$ should be considered as a function of the terminal data $(T, \xi)$. Our main idea is to let $(T, \xi)$ become "variables," and study the behavior of the value function. For notational simplicity, in this section we denote $\mathbb{L}^{2}\left(\mathcal{F}_{t}\right):=\mathbb{L}^{2}\left(\mathcal{F}_{t}, \mathbb{R}^{d^{\prime}}\right)$.

To be more precise, let us consider the following set:

$$
\mathscr{A}:=\left\{(t, \eta): t \in[0, T], \eta \in \mathbb{L}^{2}\left(\mathcal{F}_{t}\right)\right\} \subset[0, T] \times \mathbb{L}^{2}\left(\mathcal{F}_{T}\right) .
$$

We should note that the pair $(t, \eta) \in \mathscr{A}$ is "progressively measurable" in nature, that is, for each $t, \eta$ has to be $\mathcal{F}_{t}$-adapted.

We now introduce a dynamic "value" function for our original problem. Let $\Psi: \mathscr{A} \rightarrow \mathbb{R}$ be a real-valued function on $\mathscr{A}$ defined by

$$
\Psi(t, \eta)=\sup _{u \in \mathscr{U}} \varphi\left(\mathscr{Y}_{0}^{u}(t, \eta)\right), \quad(t, \eta) \in \mathscr{A} .
$$

Clearly, it holds that

$$
\Psi(0, y)=\varphi(y) \quad \text { and } \quad V_{0}(\xi)=\Psi(T, \xi) .
$$

Furthermore, we have the following easy consequences for the value function $\Psi$. Among other things, we show that a "forward" dynamic programming principle actually holds without any extra conditions, even in such a timeinconsistent setting.

Lemma 6.1. Assume that Assumption 3.1 is in force. Then:

(i) For each $t, \Psi(t, \cdot): \mathbb{L}^{2}\left(\mathcal{F}_{t}\right) \rightarrow \mathbb{R}$ is Lipschitz continuous:

$$
\left|\Psi\left(t, \eta_{1}\right)-\Psi\left(t, \eta_{2}\right)\right| \leq C\left\|\eta_{1}-\eta_{2}\right\|_{\mathbb{L}^{2}\left(\mathcal{F}_{t}\right)} \quad \text { for any } \eta_{1}, \eta_{2} \in \mathbb{L}^{2}\left(\mathcal{F}_{t}\right) .
$$

(ii) $\Psi$ satisfies the following "forward dynamic programming principle":

$$
\Psi\left(t_{2}, \eta\right)=\sup _{u \in \mathscr{U}} \Psi\left(t_{1}, \mathscr{Y}_{t_{1}}^{u}\left(t_{2}, \eta\right)\right), \quad \forall 0 \leq t_{1}<t_{2} \leq T, \eta \in \mathbb{L}^{2}\left(\mathcal{F}_{t_{2}}\right) .
$$

Proof. (i) For any $\eta_{1}, \eta_{2} \in \mathbb{L}^{2}\left(\mathcal{F}_{t}\right)$ and any $u \in \mathscr{U}$, by standard BSDE arguments we have

$$
\left|\mathscr{Y}_{0}^{u}\left(t, \eta_{1}\right)-\mathscr{Y}_{0}^{u}\left(t, \eta_{2}\right)\right|^{2} \leq C \mathbb{E}\left[\left|\eta_{1}-\eta_{2}\right|^{2}\right] .
$$

This immediately leads to (6.4) since $u \in \mathscr{U}$ is arbitrary.

(ii) Let $u \in \mathscr{U}$ be given. By the uniqueness of the BSDE, we should have

$$
\varphi\left(\mathscr{Y}_{0}^{u}\left(t_{2}, \eta\right)\right)=\varphi\left(\mathscr{Y}_{0}^{u}\left(t_{1}, \mathscr{Y}_{t_{1}}^{u}\left(t_{2}, \eta\right)\right)\right) \leq \Psi\left(t_{1}, \mathscr{Y}_{t_{1}}^{u}\left(t_{2}, \eta\right)\right) .
$$


Taking supremum over $u$, we prove " $\leq$ " part of (6.5). To see the opposite inequality, we fix an arbitrary $u \in \mathscr{U}$. For any $\varepsilon>0$, by the definition of $\Psi$, there exists $u^{\varepsilon} \in \mathscr{U}$ such that

$$
\begin{aligned}
\Psi\left(t_{1}, \mathscr{Y}_{t_{1}}^{u}\left(t_{2}, \eta\right)\right) & \leq \varphi\left(\mathscr{Y}_{0}^{u^{\varepsilon}}\left(t_{1}, \mathscr{Y}_{t_{1}}^{u}\left(t_{2}, \eta\right)\right)\right)+\varepsilon=\varphi\left(\mathscr{Y}_{0}^{u^{\varepsilon} \otimes_{t_{1}}^{u}}\left(t_{2}, \eta\right)\right)+\varepsilon \\
& \leq \Psi\left(t_{2}, \eta\right)+\varepsilon .
\end{aligned}
$$

Taking supremum over $u \in \mathscr{U}$ on the left-hand side and sending $\varepsilon$ to zero in the right-hand side, we obtain the " $\geq$ " part of (6.5) and complete the proof.

REMARK 6.2. (i) Unlike the standard DPP in stochastic control literature, (6.5) is a forward DPP in the sense that the supremum in the right-hand side acts on the smaller time $t_{1}$. This is due to the nature that our controlled dynamics is backward. This feature will also be crucial for deriving the master equation below:

(ii) In the deterministic case, the $\Psi$ here coincides with the dynamic utility $\Phi$ constructed in Section 5.1.

With the essentially "free" dynamic programming principle (6.5), it is natural to envision an HJB-type equation for the value function $\Psi$. We note that there are two fundamental differences between the current situation and the traditional ones: (i) since the DPP is "forward," the HJB equation should also be a temporally forward PDE; and (ii) since the spatial variable in the value function is now a random variable in an $\mathbb{L}^{2}$ space, which is infinite dimensional, the PDE is quite different from the traditional HJB equation (even those infinite dimensional ones), due to its adaptedness requirement on the variable $\eta$. We therefore call it a master equation, which seems to fit the situation better than a "HJB equation."

We now try to validate the idea. To begin with, we shall introduce appropriate notion of derivatives. First, for each $t \in[0, T]$, viewing $\mathbb{L}^{2}\left(\mathcal{F}_{t}\right)$ as a Hilbert space and denote by $\langle\cdot, \cdot\rangle$ its inner product, we can define the spatial derivative as the standard Fréchet derivative: for any $\eta, \tilde{\eta} \in \mathbb{L}^{2}\left(\mathcal{F}_{t}\right)$,

$$
\left\langle D_{\eta} \Psi(t, \eta), \tilde{\eta}\right\rangle:=\lim _{\varepsilon \rightarrow 0} \frac{\Psi(t, \eta+\varepsilon \tilde{\eta})-\Psi(t, \eta)}{\varepsilon},
$$

whenever the limit exists. We remark that, when $D_{\eta} \Psi(t, \eta)$ exists, it can (and will) be identified as a random variable in $\mathbb{L}^{2}\left(\mathcal{F}_{t}\right)$, thanks to the Riesz representation theorem.

The temporal derivative, however, is much more involved. We first note that the dynamic programming principle (6.5) is "forward," and more importantly, the value function is "progressive measurable," it is conceivable that there might be some difference between two directional derivatives. As it turns out, if we use the following right-temporal derivative as one often does:

$$
D_{t}^{+} \Psi(t, \eta):=\lim _{\delta \downarrow 0} \frac{\Psi(t+\delta, \eta)-\Psi(t, \eta)}{\delta}, \quad(t, \eta) \in \mathscr{A},
$$


then the corresponding master equation will become obviously ill-posed. We shall provide a detailed analysis on this point in Section 6.1 below. We will therefore use the left derivative.

A simple-minded, albeit natural, definition of the left-temporal derivative can be defined as follows:

$$
\lim _{\delta \rightarrow 0} \frac{\Psi(t, \eta)-\Psi(t-\delta, \eta)}{\delta} .
$$

However, bearing in mind the "progressive measurability" of $\Psi$ (or the definition of the set $\mathscr{A})$, we see that $\eta \in \mathbb{L}^{2}\left(\mathcal{F}_{t}\right)$ is typically not $\mathcal{F}_{t-\delta}$-measurable, so $\Psi(t-$ $\delta, \eta)$ may not even be well defined. One natural choice to overcome this issue is to modify (6.8) to the following:

$$
\lim _{\delta \rightarrow 0} \frac{\Psi(t, \eta)-\Psi\left(t-\delta, \mathbb{E}_{t-\delta}[\eta]\right)}{\delta} .
$$

However, although this definition could actually be sufficient for our purpose in this paper, it relies heavily on the underlying measure $\mathbb{P}_{0}$, which would cause many unintended consequences when we encounter situations where various probability measures are involved, as we often see in applications.

A universal, "measure-free," and potentially more applicable definition is the following "pathwise" derivative:

$$
\begin{aligned}
& D_{t}^{-} \Psi(t, \eta):=\lim _{\delta \rightarrow 0} \frac{\Psi(t, \eta)-\Psi\left(t-\delta, \eta_{t-\delta}^{t}\right)}{\delta} \\
& \text { where } \eta_{s}^{t}(\omega):=\eta\left(\omega_{s \wedge}\right),(s, \omega) \in[0, t] \times \Omega,
\end{aligned}
$$

provided the limit exists. We remark that, $D_{t}^{-} \Psi(t, \eta)$ is a real number, if it exists.

Recall Section 4.3 for the notions in pathwise analysis. We define the following.

Definition 6.3. (i) $\Psi \in C^{0}(\mathscr{A})$ if $\Psi$ is continuous in $(t, \eta)$.

(ii) $\eta \in C^{2}\left(\mathcal{F}_{t}\right)$ if the induced process $\eta^{t} \in C^{1,2}([0, t] \times \Omega)$. In this case, we denote

$$
\partial_{t} \eta:=\partial_{t} \eta_{t}^{t}, \quad \partial_{\omega} \eta:=\partial_{\omega} \eta_{t}^{t}, \quad \partial_{\omega \omega}^{2} \eta:=\partial_{\omega \omega}^{2} \eta_{t}^{t} .
$$

Moreover, denote $C_{b}^{2}\left(\mathcal{F}_{t}\right):=\left\{\eta \in C^{2}\left(\mathcal{F}_{t}\right): \eta, \partial_{t} \eta^{t}, \partial_{\omega} \eta^{t}, \partial_{\omega \omega}^{2} \eta^{t}\right.$ are bounded $\}$.

(iii) $\Psi \in C^{1}(\mathscr{A})$ if $\Psi \in C^{0}(\mathscr{A}), D_{\eta} \Psi$ exists and is in $C^{0}(\mathscr{A})$, and $D_{t}^{-} \Psi(t, \eta)$ exists for all $(t, \eta) \in \mathscr{A}_{0}$, where

$$
\mathscr{A}_{0}:=\left\{(t, \eta): 0 \leq t \leq T, \eta \in C_{b}^{2}\left(\mathcal{F}_{t}\right)\right\} \subset \mathscr{A} .
$$

We remark that, for $\Psi \in C^{0}(\mathscr{A})$, it is uniquely determined by its values in $\mathscr{A}_{0}$. 
REMARK 6.4. We should note that in general the temporal derivative of process $\eta^{t}$ [i.e., the $\operatorname{limit}_{\delta \rightarrow 0} \frac{\eta-\eta_{t-\delta}^{t}}{\delta}$ ] could fail to exist in a pathwise manner. Indeed, such limit does not exist when $\eta=B_{t}$. It is thus important to emphasize that $\Psi(t, \cdot)$ is a function on the random variable $\eta$ (on path space), rather than in pathwise sense $\Phi(t, \eta(\omega))$. As a consequence, the limit $D_{t}^{-} \Psi(t, \eta)$ does exist for many $\Psi$ and $\eta$. For example, if $\Psi(t, \eta):=\mathbb{E}[\eta]$ and $\eta=B_{t}$, then we see immediately that $\Psi\left(t-\delta, \eta_{t-\delta}^{t}\right)=\mathbb{E}\left[B_{t-\delta}\right]=0$, and thus $D_{t}^{-} \Psi\left(t, B_{t}\right)=0$.

The main result of this section is the following theorem.

THEOREM 6.5. Let Assumption 3.1 hold and $f(t, \omega, 0,0, u)$ be bounded. Assume the $\Psi$ defined by (6.2) is in $C^{1}(\mathscr{A})$. Then $\Psi$ satisfies the following master equation on $\mathscr{A}$ :

$$
\begin{cases}D_{t}^{-} \Psi(t, \eta)=\left\langle D_{\eta} \Psi(t, \eta), \partial_{t} \eta+\frac{1}{2} \operatorname{tr}\left(\partial_{\omega \omega}^{2} \eta\right)\right\rangle & \\ +\sup _{u \in \mathbb{L}^{0}\left(\mathcal{F}_{t}, U\right)}\left\langle D_{\eta} \Psi(t, \eta), f\left(t, \eta, \partial_{\omega} \eta, u\right)\right\rangle & (t, \eta) \in \mathscr{A}_{0} \\ \Psi(0, y)=\varphi(y) & y \in \mathbb{R}^{d^{\prime}}\end{cases}
$$

ProOF. Fix $0<\delta<t$. We first apply the functional Itô formula (4.18) to get $\eta_{s}^{t}=\eta-\int_{s}^{t}\left[\partial_{t} \eta_{r}^{t}+\frac{1}{2} \operatorname{tr}\left(\partial_{\omega \omega}^{2} \eta_{r}^{t}\right)\right] d r-\int_{s}^{t} \partial_{\omega} \eta_{r} \cdot d B_{r}, \quad t-\delta \leq s \leq t, \mathbb{P}_{0}$-a.s.

For any $u \in \mathscr{U}$, let $\left(\mathscr{Y}^{u}, \mathscr{Z}^{u}\right):=\left(\mathscr{Y}^{u}(t, \eta), \mathscr{Z}^{u}(t, \eta)\right)$ be the solution to BSDE (3.4). Denote

$$
\Delta Y_{s}^{u}:=\mathscr{Y}_{s}^{u}-\eta_{s}^{t}, \quad \Delta Z_{s}^{u}:=\mathscr{Z}_{s}^{u}-\partial_{\omega} \eta_{s}^{t}, \quad t-\delta \leq s \leq t .
$$

Then

$$
\begin{aligned}
\Delta Y_{s}^{u}= & \int_{s}^{t}\left[f\left(r, \mathscr{Y}_{r}^{u}, \mathscr{Z}_{r}^{u}, u_{r}\right)-\left[\partial_{t} \eta_{r}^{t}+\frac{1}{2} \operatorname{tr}\left(\partial_{\omega \omega}^{2} \eta_{r}^{t}\right)\right]\right] d r \\
& +\int_{s}^{t} \Delta Z_{r}^{u} d B_{r}, \quad t-\delta \leq s \leq t .
\end{aligned}
$$

By standard BSDE estimates we see that

$$
\mathbb{E}\left[\sup _{t-\delta \leq s \leq t}\left|\Delta Y_{s}^{u}\right|^{2}+\int_{t-\delta}^{t}\left|\Delta Z_{s}^{u}\right|^{2} d s\right] \leq C \delta^{2} .
$$

We can now apply the forward dynamic programming principle (6.5) to get

$$
\begin{aligned}
\Psi(t, \eta)-\Psi\left(t-\delta, \eta_{t-\delta}^{t}\right) & =\sup _{u \in \mathscr{U}}\left[\Psi\left(t-\delta, \mathscr{Y}_{t-\delta}^{u}\right)-\Psi\left(t-\delta, \eta_{t-\delta}^{t}\right)\right] \\
& =\sup _{u \in \mathscr{U}} \int_{0}^{1}\left\langle D_{\eta} \Psi\left(t-\delta, \eta_{t-\delta}^{t}+\theta \Delta Y_{t-\delta}^{u}\right), \Delta Y_{t-\delta}^{u}\right\rangle d \theta
\end{aligned}
$$


To identify the right-hand side above, we first deduce from (6.14) that

$$
\begin{aligned}
I_{\delta}^{u} & :=\Delta Y_{t-\delta}^{u}-\int_{t-\delta}^{t} \mathbb{E}_{t-\delta}\left[f\left(s, \eta_{s}^{t}, \partial_{\omega} \eta_{s}^{t}, u_{s}\right)-\left[\partial_{t} \eta_{s}^{t}+\frac{1}{2} \operatorname{tr}\left(\partial_{\omega \omega}^{2} \eta_{s}^{t}\right)\right]\right] d s \\
& =\int_{t-\delta}^{t} \mathbb{E}_{t-\delta}\left[f\left(s, \mathscr{Y}_{s}^{u}, \mathscr{Z}_{s}^{u}, u_{s}\right)-f\left(s, \eta_{s}^{t}, \partial_{\omega} \eta_{s}^{t}, u_{s}\right)\right] d s .
\end{aligned}
$$

Then it is not hard to check, using Assumption 3.1, that

$$
\mathbb{E}\left[\left|I_{\delta}^{u}\right|^{2}\right] \leq C \delta \mathbb{E}\left[\int_{t-\delta}^{t}\left[\left|\Delta Y_{s}^{u}\right|^{2}+\left|\Delta Z_{s}^{u}\right|^{2}\right] d s\right] \leq C \delta^{3} .
$$

Consequently, as $\delta \rightarrow 0$, we have

$$
\begin{aligned}
& \Psi(t, \eta)-\Psi\left(t-\delta, \eta_{t-\delta}^{t}\right) \\
& =\sup _{u \in \mathscr{U}}\left\langle\int_{0}^{1} D_{\eta} \Psi\left(t-\delta, \eta_{t-\delta}^{t}+\theta \Delta Y_{t-\delta}^{u}\right) d \theta,\right. \\
& \left.\int_{t-\delta}^{t} \mathbb{E}_{t-\delta}\left[f\left(s, \eta_{s}^{t}, \partial_{\omega} \eta_{s}^{t}, u_{s}\right)-\left[\partial_{t} \eta_{s}^{t}+\frac{1}{2} \operatorname{tr}\left(\partial_{\omega \omega}^{2} \eta_{s}^{t}\right)\right]\right] d s+I_{\delta}^{u}\right\rangle \\
& =\sup _{u \in \mathscr{U}}\left\langle D_{\eta} \Psi\left(t-\delta, \eta_{t-\delta}^{t}\right)\right. \text {, } \\
& \left.\int_{t-\delta}^{t} \mathbb{E}_{t-\delta}\left[f\left(s, \eta_{s}^{t}, \partial_{\omega} \eta_{s}^{t}, u_{s}\right)-\left[\partial_{t} \eta_{s}^{t}+\frac{1}{2} \operatorname{tr}\left(\partial_{\omega \omega}^{2} \eta_{s}^{t}\right)\right]\right] d s\right\rangle+o(\delta) \\
& =\sup _{u \in \mathscr{U}}\left\langle D_{\eta} \Psi\left(t-\delta, \eta_{t-\delta}^{t}\right)\right. \text {, } \\
& \left.\int_{t-\delta}^{t}\left[f\left(s, \eta_{s}^{t}, \partial_{\omega} \eta_{s}^{t}, u_{s}\right)-\left[\partial_{t} \eta_{s}^{t}+\frac{1}{2} \operatorname{tr}\left(\partial_{\omega \omega}^{2} \eta_{s}^{t}\right)\right]\right] d s\right\rangle+o(\delta) \\
& =\sup _{u \in \mathscr{U}}\left\langle D_{\eta} \Psi(t, \eta), \int_{t-\delta}^{t}\left[f\left(t, \eta, \partial_{\omega} \eta, u_{s}\right)-\left[\partial_{t} \eta+\frac{1}{2} \operatorname{tr}\left(\partial_{\omega \omega}^{2} \eta\right)\right]\right] d s\right\rangle+o(\delta) \\
& =\delta \sup _{u \in \mathbb{L}^{0}\left(\mathcal{F}_{t}, U\right)}\left\langle D_{\eta} \Psi(t, \eta), f\left(t, \eta, \partial_{\omega} \eta, u\right)-\left[\partial_{t} \eta+\frac{1}{2} \operatorname{tr}\left(\partial_{\omega \omega}^{2} \eta\right)\right]\right\rangle+o(\delta) .
\end{aligned}
$$

This implies (6.13) immediately.

REMARK 6.6. (i) From (6.13), we see that the master equation is a first-order (forward) equation (although it involves the second-order path-derivative of the state variable $\eta$ ). While this is obviously the consequence of the forward DPP (6.5) and our required initial condition on $\Psi$, it is also due to the fact that, for a forward problem, standing at $t$ and looking "left," the problem is essentially "deterministic," hence the corresponding "HJB" equation should be first-order. The left-temporal path derivative that we introduced in (6.10) is thus essential. 
(ii) The main difficulty of this approach is the proper solution of the master equation (6.13). To the best of our knowledge, such an equation is completely new in the literature. Its well-posedness, in strong, weak and viscosity sense, seem to be all open at this point. We hope to be able to address some of them in our future research.

6.1. An ill-posed master equation. We have emphasized at above the importance of using the left-temporal derivative, given the fact that $\Psi$ satisfies a forward dynamic programming principle. In what follows, we shall reinforce this point by explaining how a "traditional" right-temporal derivative (6.7) could actually lead to an ill-posed master equation. We first note that, since by our definition of $\mathscr{A}$, for each $\delta>0, \eta \in \mathbb{L}^{2}\left(\mathcal{F}_{t}\right) \subset \mathbb{L}^{2}\left(\mathcal{F}_{t+\delta}\right)$, thus $\Psi(t+\delta, \eta)$ is well defined for all $(t, \eta) \in \mathscr{A}$.

Now let us derive the equation for the $\Psi$ in (6.2) involving such a derivative. Again, by DPP (6.5) we have

$$
\begin{aligned}
\Psi(t+\delta, \eta)-\Psi(t, \eta) & =\sup _{u \in \mathscr{U}}\left[\Psi\left(t, \mathscr{Y}_{t}^{u}(t+\delta, \eta)\right)-\Psi(t, \eta)\right] \\
& =\sup _{u \in \mathscr{U}} \int_{0}^{1}\left\langle D_{\eta} \Psi\left(t, \eta+\theta \mathcal{Y}_{t}^{u}\right), \mathcal{Y}_{t}^{u}\right\rangle d \theta
\end{aligned}
$$

where $\mathcal{Y}_{s}^{u}:=\mathscr{Y}_{s}^{u}(t+\delta, \eta)-\eta, t \leq s \leq t+\delta$. Note that, if we denote $\mathcal{Z}_{s}^{u}:=$ $\mathscr{Z}_{s}^{u}(t+\delta, \eta)$, then $\left(\mathcal{Y}^{u}, \mathcal{Z}^{u}\right)$ satisfies the BSDE

$$
\mathcal{Y}_{s}^{u}=\int_{s}^{t+\delta} f\left(r, \eta+\mathcal{Y}_{r}^{u}, \mathcal{Z}_{r}^{u}, u_{r}\right) d r-\int_{s}^{t+\delta} \mathcal{Z}_{r}^{u} d B_{r}, \quad t \leq s \leq t+\delta
$$

Then the standard BSDE estimates would tell us that

$$
\mathbb{E}\left[\sup _{t \leq s \leq t+\delta}\left|\mathcal{Y}_{s}^{u}\right|^{2}+\int_{t}^{t+\delta}\left|\mathcal{Z}_{s}^{u}\right|^{2} d s\right] \leq C \delta^{2} .
$$

Again, let us denote

$$
I_{\delta}^{u}:=\mathcal{Y}_{t}^{u}-\mathbb{E}_{t}\left[\int_{t}^{t+\delta} f\left(s, \eta, 0, u_{s}\right) d s\right] .
$$

Then, assuming Assumption 3.1 we have

$$
\begin{aligned}
\left|I_{\delta}^{u}\right| & =\left|\mathbb{E}_{t}\left[\int_{t}^{t+\delta}\left[f\left(s, \eta+\mathcal{Y}_{s}^{u}, \mathcal{Z}_{s}^{u}, u_{s}\right)-f\left(s, \eta, 0, u_{s}\right)\right] d s\right]\right| \\
& \leq C \mathbb{E}_{t}\left[\int_{t}^{t+\delta}\left[\left|\mathcal{Y}_{s}^{u}\right|+\left|\mathcal{Z}_{s}^{u}\right|\right] d s\right]
\end{aligned}
$$

and consequently

$$
\mathbb{E}\left[\left|I_{\delta}^{u}\right|^{2}\right] \leq C \delta \mathbb{E}\left[\int_{t}^{t+\delta}\left[\left|\mathcal{Y}_{s}^{u}\right|^{2}+\left|\mathcal{Z}_{s}^{u}\right|^{2}\right] d s\right] \leq C \delta^{3}
$$


Now (6.15) will lead to

$$
\begin{aligned}
\Psi(t+\delta, \eta)-\Psi(t, \eta) & =\sup _{u \in \mathscr{U}}\left\langle D_{\eta} \Psi(t, \eta), \mathbb{E}_{t}\left[\int_{t}^{t+\delta} f\left(s, \eta, 0, u_{s}\right) d s\right]\right\rangle+o(\delta) \\
& =\delta \sup _{u \in \mathbb{L}^{2}\left(\mathcal{F}_{t}, U\right)}\left\langle D_{\eta} \Psi(t, \eta), f(t, \eta, 0, u)\right\rangle+o(\delta) .
\end{aligned}
$$

In other words, we will arrive at the following first-order PDE:

$$
\left\{\begin{array}{l}
D_{t}^{+} \Psi(t, \eta)=\sup _{u \in \mathbb{L}^{2}\left(\mathcal{F}_{t}, U\right)}\left\langle D_{\eta} \Psi(t, \eta), f(t, \eta, 0, u)\right\rangle \quad(t, \eta) \in \mathscr{A} ; \\
\Psi(0, y)=\varphi(y) .
\end{array}\right.
$$

We remark that equation (6.16) is typically ill-posed. Indeed, (6.16) involves only $f(\cdot, \cdot, 0, \cdot)$, while the $\Psi$ defined in (6.2) obviously depends on $f(\cdot, \cdot, z, \cdot)$. So unless the function $f$ is independent of the variable $z$, there is essentially no hope that equation (6.16) will have a unique solution, as the value functions of two completely different optimization problems can satisfy the same master equation. We therefore conclude that $D_{t}^{-} \Psi$, not $D_{t}^{+} \Psi$, is the right choice of temporal derivative for the master equation.

\section{REFERENCES}

[1] Aubin, J.-P. and Frankowska, H. (2009). Set-Valued Analysis. Birkhäuser, Boston, MA. MR2458436

[2] BJORK, T. and MURGoci, A. (2010). A general theory of markovian time inconsistent stochastic control problems. Available at ssrn.com/abstract=1694759.

[3] BJörk, T., Murgoci, A. and Zhou, X. Y. (2014). Mean-variance portfolio optimization with state-dependent risk aversion. Math. Finance 24 1-24. MR3157686

[4] Bouchard, B., Elie, R. and TouzI, N. (2009/10). Stochastic target problems with controlled loss. SIAM J. Control Optim. 48 3123-3150. MR2599913

[5] Cardaliaguet, P., Delarue, F., Lasry, J. M. and Lions, P. L. (2015). The master equation and the convergence problem in mean field games. Preprint. Available at arXiv:1509.02505.

[6] Cohen, S. and Elliott, R. (2009). Time consistency and moving horizons for risk measures. Preprint. Available at arXiv:0912.1396.

[7] Cont, R. and Fournié, D.-A. (2013). Functional Itô calculus and stochastic integral representation of martingales. Ann. Probab. 41 109-133. MR3059194

[8] CUI, X., LI, D., WAng, S. and ZHU, S. (2012). Better than dynamic mean-variance: Time inconsistency and free cash flow stream. Math. Finance 22 346-378. MR2897388

[9] Cvitanić, J. and Zhang, J. (2013). Contract Theory in Continuous-Time Models. Springer, Heidelberg. MR2963805

[10] DupiRe, B. (2009). Functional Itô calculus. Preprint. Available at papers.ssrn.com.

[11] EKELAND, I. and LAZRAK, A. (2010). The golden rule when preferences are time inconsistent. Math. Financ. Econ. 4 29-55. MR2746576

[12] Ekren, I., Keller, C., Touzi, N. and Zhang, J. (2014). On viscosity solutions of path dependent PDEs. Ann. Probab. 42 204-236. MR3161485 
[13] Ekren, I., TouzI, N. and ZHANG, J. (2016). Viscosity solutions of fully nonlinear parabolic path dependent PDEs: Part I. Ann. Probab. 44 1212-1253.

[14] EKREN, I., TOUZI, N. and ZHANG, J. (2016). Viscosity solutions of fully nonlinear parabolic path dependent PDEs: Part II. Ann. Probab. 44 2507-2553. MR3531674

[15] EkREN, I., TOUZI, N. and ZHANG, J. (2016). Viscosity solutions of fully nonlinear parabolic path dependent PDEs: Part I. Ann. Probab. 44 1212-1253. MR3474470

[16] Ekren, I. and ZhANG, J. (2016). Pseudo-Markovian viscosity solutions of fully nonlinear degenerate PPDEs. Probab. Uncertain. Quant. Risk 1 Paper No. 6, 34. MR3583183

[17] El Karoui, N. and Mrad, M. (2013). An exact connection between two solvable SDEs and a nonlinear utility stochastic PDE. SIAM J. Financial Math. 4 697-736. MR3106475

[18] Feinstein, Z. and RudlofF, B. (2013). Time consistency of dynamic risk measures in markets with transaction costs. Quant. Finance 13 1473-1489. MR3175918

[19] Feinstein, Z. and Rudloff, B. (2016). Time consistency for scalar multivariate risk measures. Working paper.

[20] HU, Y., JiN, H. and ZHOU, X. Y. (2012). Time-inconsistent stochastic linear-quadratic control. SIAM J. Control Optim. 50 1548-1572. MR2968066

[21] HU, Y. and PENG, S. (2006). On the comparison theorem for multidimensional BSDEs. C. $R$. Math. Acad. Sci. Paris 343 135-140. MR2243308

[22] Kahneman, D. and Tversky, A. (1979). Prospect theory: An analysis of decision under risk. Econometrica 47 263-292.

[23] Kahneman, D. and Tversky, A. (1992). Advances in prospect theory: Cumulative representation of uncertainty. J. Risk and Uncertainty 5 297-323.

[24] Keller, C. and ZHANG, J. (2016). Pathwise Itô calculus for rough paths and rough PDEs with path dependent coefficients. Stochastic Process. Appl. 126 735-766. MR3452811

[25] Kydland, F. and PRescotT, E. (1977). Rules rather than discretion: The inconsistency of optimal plans. J. Polit. Econ. 85 473-492.

[26] MA, J. and YonG, J. (1995). Solvability of forward-backward SDEs and the nodal set of Hamilton-Jacobi-Bellman equations. Chin. Ann. Math. Ser. B 16 279-298. [A Chinese summary appears in Chinese Ann. Math. Ser. A 16 (1995), no. 4, 532.] MR1370779

[27] Miller, C. W. (2017). Nonlinear PDE approach to time-inconsistent optimal stopping. SIAM J. Control Optim. 55 557-573. MR3614676

[28] Musiela, M. and Zariphopoulou, T. (2007). Investment and valuation under backward and forward dynamic exponential utilities in a stochastic factor model. In Advances in Mathematical Finance. Appl. Numer. Harmon. Anal. 303-334. Birkhäuser, Boston, MA. MR2359374

[29] Musiela, M. and Zariphopoulou, T. (2010). Stochastic partial differential equations and portfolio choice. In Contemporary Quantitative Finance 195-216. Springer, Berlin. MR2732847

[30] REN, Z. and TAN, X. (2017). On the convergence of monotone schemes for path-dependent PDEs. Stochastic Process. Appl. 127 1738-1762. MR3646429

[31] Soner, H. M. and TouzI, N. (2002). Dynamic programming for stochastic target problems and geometric flows. J. Eur. Math. Soc. (JEMS) 4 201-236. MR1924400

[32] Strotz, R. H. (1955). Myopia and inconsistency in dynamic utility maximization. Rev. Econ. Stud. 23 165-180.

[33] XU, Z. Q. and Zhou, X. Y. (2013). Optimal stopping under probability distortion. Ann. Appl. Probab. 23 251-282. MR3059235

[34] Yong, J. (2012). Time-inconsistent optimal control problems and the equilibrium HJB equation. Math. Control Relat. Fields 2 271-329. MR2991570

[35] ZhANG, J. and ZHUO, J. (2014). Monotone schemes for fully nonlinear parabolic path dependent PDEs. J. Financ. Eng. 1 1450005. (23 pages). 
[36] ZHou, X. Y. (2010). Mathematicalising behavioural finance. In Proceedings of the International Congress of Mathematicians. Volume IV 3185-3209. Hindustan Book Agency, New Delhi. MR2828011

\author{
DEPARTMENT OF MATHEMATICS \\ UNIVERSITY OF SOUTHERN CALIFORNIA \\ LOS ANGELES, CALIFORNIA 90089 \\ USA \\ E-MAIL: chandrasekhar.karnam@gmail.com \\ jinma@usc.edu \\ jianfenz@usc.edu
}

